\title{
Wadge hardness in Scott spaces and its effectivization
}

\author{
VERÓNICA BECHER ${ }^{\ddagger}, \dagger$ and SERGE GRIGORIEFF $F^{\dagger, \S}$ \\ ${ }^{\ddagger}$ FCEyN,Universidad de Buenos Aires \& CONICET, Argentina \\ Email: vbecher@dc.uba.ar \\ ${ }^{\S}$ LIAFA, CNRS \& Université Paris Diderot - Paris 7, France \\ Email: seg@liafa.univ-paris-diderot.fr
}

Received 7 March 2012

\begin{abstract}
We prove some results on the Wadge order on the space of sets of natural numbers endowed with Scott topology, and more generally, on omega-continuous domains. Using alternating decreasing chains we characterize the property of Wadge hardness for the classes of the Hausdorff difference hierarchy (iterated differences of open sets). A similar characterization holds for Wadge one-to-one and finite-to-one completeness. We consider the same questions for the effectivization of the Wadge relation. We also show that for the space of sets of natural numbers endowed with the Scott topology, in each class of the Hausdorff difference hierarchy there are two strictly increasing chains of Wadge degrees of sets properly in that class. The length of these chains is the rank of the considered class, and each element in one chain is incomparable with all the elements in the other chain.
\end{abstract}

\section{Introduction}

Wadge reducibility between subsets of possibly uncountable topological spaces is the topological version of the classical many-one reducibility between subsets of discrete countable spaces like words or integers. Since Wadge reducibility defines a preorder on subsets of the topological space, one can consider the associated equivalence relation, whose classes are called Wadge degrees. The theory of Wadge degrees is remarkable in the case of Polish (i.e. completely metrizable with some countable dense subset) zerodimensional (i.e. admitting some basis of clopen sets) topological spaces, for instance the Cantor space $\mathbf{2}^{\omega}$ and the Baire space $\mathbb{N}^{\omega}$ with the product of discrete topologies. Wadge (1972) developed such theory by looking at reductions between subsets of $\mathbb{N}^{\omega}$ as winning strategies in adequate infinite games, the now so-called Wadge games. The strength of Wadge's theory comes from Martin's result (1975) about determinacy of Borel games. The Wadge order on Borel subsets of $\mathbb{N}^{\omega}$ is well founded and almost total: every antichain has only two elements, and it is constituted by the Wadge degree of a set and that of its complement. In spaces that are not totally disconnected this beauty breaks down. For instance, in the real line $\mathbb{R}$, the Wadge order is not well founded (Hertling

\footnotetext{
† Members of the Laboratoire Internationale Associé INFINIS, Universidad de Buenos Aires-Université Paris Diderot-Paris 7. This research was partially done whilst the first author was a visiting fellow at the Isaac Newton Institute for Mathematical Sciences in the programme 'Semantics \& Syntax'.
} 
1996a;1996b), for each $2 \leq \xi<\omega_{1}$ there are chains of proper $\boldsymbol{\Sigma}_{\xi}^{0}$ sets indexed by any countable ordinal (Ikegami 2010) and, letting $\subseteq^{*}$ denote inclusion up to a finite set, the preorder $\left(\mathcal{P} \mathbb{N}, \subseteq^{*}\right)$ (hence also any partial order of cardinality $\aleph_{1}$ ) can be embedded in the Wadge preorder of Borel subsets of $\mathbb{R}$ (Ikegami et al. 2012). Also in every metric space which is positive-dimensional (i.e. at some point there is no clopen local base), there are $2^{\aleph_{0}}$ Wadge incomparable sets in the Hausdorff class $\mathbf{D}_{2}$ of differences of two open sets (Schlicht 2012).

Outside zero-dimensional Polish spaces, Wadge theory has been first considered in the space $\mathcal{P} \mathbb{N}$ of sets of natural numbers endowed with the Scott topology (which is $T_{0}$ but not $T_{2}$, i.e. not Hausdorff), by Tang $(1979 ; 1981)$. Then it has been extensively studied by Selivanov in a series of papers (Selivanov 2004; 2005a; 2005b; 2006; 2008 and references therein) in the context of $\omega$-continuous domains. Although much of Wadge theory fails in spaces with Scott topology, a non-trivial part remains. For instance, Selivanov showed the nonexistence of self-dual degrees in the Hausdorff difference hierarchy.

Another problem is to understand what part of Wadge theory remains in effective topological spaces by considering effective reductions. Since Borel determinacy gives highly non-computable game strategies, the main tool used in the classical Wadge theory is not available. As pointed out to us by an anonymous referee, it follows from Fokina et al. (2010) that the structure of effective Wadge degrees is very different, even for the Baire space: the ordered structure of inclusion on computably enumerable subsets of $\mathbb{N}$ can be embedded into the effective Wadge hierarchy of $\Sigma_{2}^{0}$ subsets of the Baire space; cf. Proposition 3.8 infra.

In this paper, we concentrate on the Wadge theory on the classes of the Hausdorff difference hierarchy, i.e. on $\Delta_{2}^{0}$ Borel sets. We also consider the same problems but using only effective reductions. With this study, we provide the general framework for the particular cases of effective Wadge hardness we used to define highly random reals in Becher and Grigorieff $(2005 ; 2009)$. The paper is organized as follows. Section 2 recalls the basic definitions of the Borel and Hausdorff hierarchies and some material on domains and effective versions of various notions. In Section 3, we present Wadge reductions in general topological spaces, and their effective versions. We make the relation between Wadge completeness and universality explicit, and we recall what is already known about the Wadge theory outside zero-dimensional spaces. In Section 4, we prove the main results of the paper. Theorem 4.8 characterizes Wadge hardness for the classes of the Hausdorff difference hierarchy in terms of alternating decreasing chains. Theorem 4.14 gives a similar characterization for Wadge one-to-one and finite-to-one completeness. We prove effective versions of these results.

Theorem 4.23 shows that, for the space of sets of natural numbers endowed with the Scott topology, in each class of the Hausdorff hierarchy there are two strictly increasing chains of Wadge degrees of sets properly in that class. The length of these chains is the rank of the considered class, and each element in one chain is incomparable with all the elements in the other chain. This result builds on the construction done by Selivanov (2005b) of two incomparable Wadge degrees which properly belong to an arbitrarily given Hausdorff class. Finally in Section 5 we prove results on the Wadge theory for the Borel class $\Sigma_{2}^{0}$. 


\section{Preliminaries}

For any set $A, \mathcal{P} A$ denotes the powerset of $A$ and $\mathcal{P}_{<\omega} A$ denotes the family of all finite subsets of $A$. We write $\mathbb{N}$ for the set of natural numbers. When $X$ is a subset of $\mathbb{N}$, and $i \in \mathbb{N}, X+i=\{n+i \mid n \in X\}$ and $i X=\{i n \mid n \in X\}$. Subsets of $\mathbb{N}$ are simply called sets, subsets of $\mathcal{P} \mathbb{N}$ are called families, and collections of subsets of $\mathcal{P} \mathbb{N}$ are called classes. As usual, $\mathbb{N}^{\omega}$ is the set of infinite sequences of natural numbers (the Baire space), and $\mathbb{Z}, \mathbb{Q}, \mathbb{R}$ denote the sets of integer, rational and real numbers, respectively. Greek letters $\alpha, \beta, \gamma, \delta$ are used to denote ordinals. We write $\omega$ for the first infinite ordinal, $\omega_{1}$ for the first uncountable ordinal, and $\omega_{1}^{C K}$ for the least non-computable ordinal (the Church-Kleene ordinal). For any two ordinals $\alpha, \beta, \alpha \sim \beta$ means that they have the same parity.

\subsection{Borel and Hausdorff hierarchies}

Following Selivanov (2005b; 2008), we consider the Borel hierarchy and the HausdorffKuratowski difference hierarchy in a general topological space $P$. The Borel hierarchy consists of classes of subsets of $P$, namely $\boldsymbol{\Sigma}_{\alpha}^{0}, \boldsymbol{\Pi}_{\alpha}^{0}, \boldsymbol{\Delta}_{\alpha}^{0}$, where $1 \leq \alpha<\omega_{1}$. They are defined by induction on $\alpha: \boldsymbol{\Sigma}_{1}^{0}$ is the class of open subsets of $P, \boldsymbol{\Sigma}_{2}^{0}$ is the class of countable unions of differences of open subsets of $P$, and, for $\alpha>2, \Sigma_{\alpha}^{0}$ is the class of countable unions of sets in $\bigcup_{\beta<\alpha} \boldsymbol{\Pi}_{\beta}^{0}$. The class $\boldsymbol{\Pi}_{\alpha}^{0}$ is the class of complements of sets in $\boldsymbol{\Sigma}_{\alpha}^{0}$ and $\boldsymbol{\Delta}_{\alpha}^{0}=\boldsymbol{\Sigma}_{\alpha}^{0} \cap \boldsymbol{\Pi}_{\alpha}^{0}$. The class $\mathbf{G}_{\delta}$ (respectively $\mathbf{F}_{\sigma}$ ) is the family of countable intersections of open sets (respectively countable unions of closed sets). In general, it is a proper subclass of $\boldsymbol{\Pi}_{2}^{0}$ (respectively $\boldsymbol{\Sigma}_{2}^{0}$ ).

For an ordinal $1 \leq \alpha<\omega_{1}$, the operation $D_{\alpha}$ sends an $\alpha$-sequence of sets $\left(A_{\beta}\right)_{\beta<\alpha}$ to the set $D_{\alpha}\left(\left(A_{\beta}\right)_{\beta<\alpha}\right)=\bigcup\left\{A_{\beta} \backslash \cup_{\gamma<\beta} A_{\gamma} \mid \beta<\alpha, \beta \psi \alpha\right\}$ (recall that $\beta \psi \alpha$ means that $\alpha, \beta$ have different parities). The Hausdorff hierarchy is constituted by the Hausdorff classes $\mathbf{D}_{\alpha}, c o$ - $\mathbf{D}_{\alpha}$ where $1 \leq \alpha<\omega_{1}$. The class $\mathbf{D}_{\alpha}$ consists of all subsets of $P$ of the form $D_{\alpha}\left(\left(A_{\beta}\right)_{\beta<\alpha}\right)$, where $\left(A_{\beta}\right)_{\beta<\alpha}$ is an $\alpha$-sequence of open subsets of $P$ (with no loss of generality, this $\alpha$-sequence can be supposed increasing with respect to the inclusion relation). The class $c o-\mathbf{D}_{\alpha}$ is the class of complements of sets in $\mathbf{D}_{\alpha}$. The Hausdorff-Kuratowski hierarchy is obtained by replacing open sets by sets in a $\boldsymbol{\Sigma}_{\xi}^{0}$ class, $\xi<\omega_{1}$ : its classes are denoted by $\mathbf{D}_{\alpha}\left(\boldsymbol{\Sigma}_{\xi}^{0}\right)$ and co-D $\mathbf{D}_{\alpha}\left(\boldsymbol{\Sigma}_{\xi}^{0}\right)$. Hausdorff-Kuratowski theorem ensures that $\bigcup_{1 \leq \alpha<\omega_{1}} \mathbf{D}_{\alpha}\left(\boldsymbol{\Sigma}_{\xi}^{0}\right)=\boldsymbol{\Delta}_{\xi+1}^{0}$ for any $1 \leq \xi<\omega_{1}$.

When the space $P$ is not obvious from the context, or when we want to make it explicit, we will add a parenthesized reference to the space $P$, writing $\boldsymbol{\Sigma}_{\alpha}^{0}(P), \boldsymbol{\Pi}_{\alpha}^{0}(P), \boldsymbol{\Delta}_{\alpha}^{0}(P)$, $\mathbf{G}_{\delta}(P), \mathbf{F}_{\sigma}(P), \mathbf{D}_{\alpha}(P), \mathbf{D}_{\alpha}\left(\Sigma_{\xi}^{0}(P)\right), c o-\mathbf{D}_{\alpha}\left(\Sigma_{\xi}^{0}(P)\right)$. Let us mention the following classical result.

Fact 2.1. If $f: P \rightarrow Q$ is continuous then the inverse image of a subset of $Q$ in some Borel or Hausdorff-Kuratowski class of $Q$ is in the corresponding class of subsets of $P$.

\subsection{Domains}

We briefly recall the main definitions and refer the reader to classical papers and books, for instance (Abramsky and Jung 1994; Edalat 1997; Gierz et al. 2003). A directed 
complete partial order (dcpo) is a partially ordered set $(P, \sqsubseteq$ ) such that every nonempty directed subset $S$ has a least upper bound (denoted by $\sqcup S$ ). A subset $X$ of $P$ is an upset (respectively downset) if, for all $x, y \in P$, if $x \in X$ and $x \sqsubseteq y$ (respectively $y \sqsubseteq x$ ) then $y \in X$. The Scott topology on a dcpo $P$ admits as closed sets all downsets closed under suprema of directed subsets of $P$. Thus, open subsets are upsets $O$ such that every directed set with supremum in $O$ has an element in $O$. The Scott topology is $T_{0}$ (if $x \neq y$ then there exists an open set which contains only one of the two points $x, y$ ). The order relation $\subseteq$ can be recovered from the Scott topology as the specialization order: $x \subseteq y$ if and only if every open set which contains $x$ also contains $y$. A function $f: P \rightarrow Q$ between two depo's is continuous with respect to the Scott topologies if and only if it is increasing and preserves suprema of directed subsets: if $S \subseteq P$ is directed then $f(\sqcup S)=\sqcup f(S)$.

The approximation (or way-below) relation in a dcpo $(P$, 드) is defined as follows: $x \ll y$ if, for all directed subsets $S, y \sqsubseteq \sqcup S$ implies $x \sqsubseteq s$ for some $s \in S$. Thus, $x \ll y \Rightarrow x \sqsubseteq y$ and $x^{\prime} \sqsubseteq x \ll y \sqsubseteq y^{\prime} \Rightarrow x^{\prime} \ll y^{\prime}$. An element $x \in P$ is compact (or finite) if $x \ll x$. If $x$ is compact then $x \ll y \Leftrightarrow x \sqsubseteq y$. The set of compact elements is denoted $K(P)$. A continuous domain is a dcpo such that, for every $x \in P$, the set $\downarrow x=\{z \in P \mid z \ll x\}$ is directed and $x=\sqcup \downarrow x$. A basis of a continuous domain is any set $B$ such that, for all $x, B \cap \downarrow x$ is directed and $x=\sqcup(B \cap \downarrow x)$. An $\omega$-continuous domain is a dcpo admitting a countable basis. An $\omega$-algebraic domain is a depo for which $K(P)$ is a countable basis. If $B$ is a basis of a continuous domain then the sets $\uparrow b$, for $b \in B$, form a topological basis, where $\uparrow x=\{y \mid x \ll y\}$. Let us mention the classical interpolation property.

Fact 2.2. Let $B$ be a basis of the continuous domain $P$. Suppose $y \in P$ and $X \subseteq P$ is finite such that for each $x \in X, x \ll y$. Then, there exists $b \in B$ such that for each $x \in X \quad x \ll b$ and $b \ll y$.

\subsection{The Scott domain $\mathcal{P} \mathbb{N}$}

The ordered set $(\mathcal{P N}, \subseteq)$ is an $\omega$-algebraic domain, its compact elements are the finite sets and $X \ll Y$ if and only if $X$ is finite and included in $Y$. A topological basis of the Scott topology is the class $\left\{\mathcal{B}_{A} \mid A \in \mathcal{P}_{<\omega} \mathbb{N}\right\}$ where $\mathcal{B}_{A}=\{X \in \mathcal{P} \mathbb{N} \mid A \subseteq X\}$. The Scott topology gives 'positive information' about sets, and contrasts with the Cantor topology on $\mathbf{2}^{\omega}$ which gives positive and negative information about sets via their characteristic functions. As recalled before, the Scott topology on $\mathcal{P} \mathbb{N}$ is $T_{0}$ : if $i \in X \backslash Y$, the open set $\mathcal{B}_{\{i\}}$ contains $X$ but not $Y$.

As observed by Selivanov (2005b) the classes of finite rank of the Borel hierarchy in $\mathcal{P}$ do not coincide with the corresponding ones in the Cantor space $\mathbf{2}^{\omega}$ : for all $n \in \mathbb{N}$, $n \geq 1, \boldsymbol{\Sigma}_{n}^{0}(\mathcal{P} \mathbb{N}) \varsubsetneqq \boldsymbol{\Sigma}_{n}^{0}\left(\mathbf{2}^{\omega}\right) \varsubsetneqq \boldsymbol{\Sigma}_{n+1}^{0}(\mathcal{P} \mathbb{N})$. For instance, $\mathcal{X}=\mathcal{P} \mathbb{N} \backslash\{\mathbb{N}\}$, defined by the formula $\exists x(x \notin X)$, is $\boldsymbol{\Sigma}_{1}^{0}\left(\mathbf{2}^{\omega}\right)$ and $\boldsymbol{\Sigma}_{2}^{0}(\mathcal{P} \mathbb{N})$ but neither Scott open nor Scott closed. However, the classes of infinite rank of the Borel hierarchy in $\mathcal{P N}$ and $\mathbf{2}^{\omega}$ coincide. The only subfamilies of $\mathcal{P} \mathbb{N}$ which are both open and $\mathbf{F}_{\sigma}$ are $\varnothing$ and $\mathcal{P} \mathbb{N}$. To see why, suppose $\mathcal{O}$ is open, $\mathcal{O} \neq \varnothing$, and $\mathcal{X}$ is $\mathbf{F}_{\sigma}, \mathcal{X} \neq \mathcal{P} \mathbb{N}$. Let $\mathcal{X}=\bigcup_{i \in \mathbb{N}}\left(\mathcal{P} \mathbb{N} \backslash \mathcal{O}_{i}\right)$ where $\mathcal{O}_{i}$ is open. Observe that $\mathcal{O}_{i}$ is nonempty. Choose finite sets $A$ and $B_{i}$, for $i \in \mathbb{N}$, such that $\mathcal{B}_{A} \subseteq \mathcal{O}$ and $\mathcal{B}_{B_{i}} \subseteq \mathcal{O}_{i}$. Let $C=A \cup \bigcup_{i \in \mathbb{N}} B_{i}$. Then $C \in \mathcal{O}$ since $C \supseteq A$ and $C \in \mathcal{O}_{i}$ since $C \supseteq B_{i}$. Thus, 
$C$ is in $\mathcal{O} \backslash \mathcal{X}$, showing $\mathcal{O} \neq \mathcal{X}$. A map $f: \mathcal{P} \rightarrow \mathcal{P} \mathbb{N}$ is Scott continuous if and only if $f(X)=\bigcup_{A \subseteq X, A \in \mathcal{P}_{<\omega} \mathbb{N}} f(A)$ for all $X$. If $f: \mathcal{P} \mathbb{N} \rightarrow \mathcal{P} \mathbb{N}$ is a continuous bijection then there exists a permutation $\theta: \mathbb{N} \rightarrow \mathbb{N}$ such that for all $X, f(X)=\{\theta(x) \mid x \in X\}$. In particular, $f^{-1}$ is also continuous. Indeed, the inclusion order is a topological notion (namely, the specialization order) hence $f$ is an automorphism of $(\mathcal{P N}, \subseteq)$ and as such, respects unions and maps singleton sets into singleton sets. Let us mention a classical result which shows that $\mathcal{P} \mathbb{N}$ is 'universal' for $\omega$-continuous domains.

Fact 2.3. Let $\left(b_{n}\right)_{n \in \mathbb{N}}$ enumerate a countable basis of the $\omega$-continuous domain $(P, \subseteq)$. Let $\varphi: P \rightarrow \mathcal{P N}$ be such that $\varphi(x)=\left\{n \mid b_{n} \ll x\right\}$.

1. The map $\varphi$ is a dcpo embedding (i.e. an isomorphism between $(P, \subseteq)$ and $(\varphi(P), \subseteq)$ such that $\{x \in P \mid \varphi(x) \subseteq Z\}$ is directed for any $Z \in \mathcal{P} \mathbb{N})$.

2. The map $\varphi$ is is a topological embedding of $P$ into $\mathcal{P N}$ (i.e. an homeomorphism from $P$ to the subspace $\varphi(P)$ of $\mathcal{P} \mathbb{N})$. Then, for any Borel or Hausdorff-Kuratowski class $\boldsymbol{\Gamma},\{\varphi(Z) \mid Z \in \boldsymbol{\Gamma}(P)\}=\boldsymbol{\Gamma}(\varphi(P))=\{\mathcal{X} \cap \varphi(P) \mid \mathcal{X} \in \boldsymbol{\Gamma}(\mathcal{P} \mathbb{N})\}$.

3. $\varphi(P)$ is $\Pi_{2}^{0}$ in $\mathcal{P} \mathbb{N}$.

Proof. We just prove items 2 and 3. Since $\varphi$ is injective, it suffices to consider the case of basic open sets $\uparrow b_{n}$ 's of $P$. Observe that $\varphi\left(\uparrow b_{n}\right)=\left\{\varphi(x) \mid x \in P, b_{n} \ll x\right\}=\mathcal{B}_{\{n\}} \cap \varphi(P)$. This proves item 2. For item 3 observe that $X \in \varphi(P)$ if and only if, relative to the approximation relation $\ll$ of $P,\left\{b_{i} \mid i \in X\right\}$ is a directed downset. Letting $R=\left\{(i, j) \mid b_{i} \ll b_{j}\right\}$, this means that $X$ satisfies the formulas $\forall i, j \exists k(i, j \in X \Rightarrow(k \in X \wedge(i, k) \in R \wedge(j, k) \in R))$ and $\forall i, j(j \in X \wedge(i, j) \in R \Rightarrow i \in X)$, i.e., $X \in \varphi(P)$ if and only if $X$ is in the $\Pi_{2}^{0}$ families $\bigcap_{i, j \in \mathbb{N}} \cup_{k \in Z_{i, j}}\left(\mathcal{B}_{\{k\}} \cup\left(\mathcal{P} \mathbb{N} \backslash \mathcal{B}_{\{i, j\}}\right)\right)$ and $\bigcap_{(i, j) \in R}\left(\mathcal{B}_{\{i\}} \cup\left(\mathcal{P N} \backslash \mathcal{B}_{\{j\}}\right)\right)$, where $Z_{i, j}=\{k \mid(i, k) \epsilon$ $R$ and $(j, k) \in R\}$.

Remark 2.4. Fact 2.3 allows us to transfer properties from $\mathcal{P} \mathbb{N}$ to $\omega$-continuous domains.

\subsection{Effectivization}

2.4.1. Presentations of topological spaces. The following definition slightly departs from Weihrauch (2000, page 63).

Definition 2.5. Let $P, Q$ be $T_{0}$ second-countable topological spaces.

1. A presentation of the topology of $P$ is an enumeration (not necessarily injective) $\left(U_{n}\right)_{n \in \mathbb{N}}$ of some topological basis of $P$. For convenience, we shall sometimes consider enumerations indexed by $\mathbb{N}^{2}$ or $\mathcal{P}_{<\omega} \mathbb{N}$.

2. A presentation $\left(U_{n}\right)_{n \in \mathbb{N}}$ is effective if $\left\{(i, j) \mid U_{i} \subseteq U_{j}\right\}$ is computably enumerable. An effective topological space consists of a $T_{0}$ second-countable topological space $P$ together with an effective presentation of $P$.

3. Relative to a presentation $\left(U_{n}\right)_{n \in \mathbb{N}}$ of the topology of $P$, an element $x$ of $P$ is effective (respectively computable) if the set $\left\{i \in \mathbb{N} \mid x \in U_{i}\right\}$ is computably enumerable (respectively computable). Let $\alpha<\omega_{1}^{C K}$ be an infinite computable ordinal. A sequence $\left(x_{\beta}\right)_{\beta<\alpha}$ of elements of $P$ is effective (respectively computable) if there exists a computable relation $R \subseteq \mathbb{N}^{2}$, isomorphic to the ordering of $\alpha$, such that, letting 
$\rho: \mathbb{N} \rightarrow \alpha$ be the isomorphism between $(\mathbb{N}, R)$ and $(\alpha,<)$, the relation $\left\{(n, i) \mid x_{\rho(n)} \in U_{i}\right\}$ is computably enumerable (respectively computable). In that case, each element of the $\alpha$-sequence is effective (respectively computable).

4. Relative to presentations $\left(U_{n}\right)_{n \in \mathbb{N}},\left(V_{n}\right)_{n \in \mathbb{N}}$ of the topologies of $P$ and $Q$, a continuous map $f: P \rightarrow Q$ is effective if the set $\left\{(i, j) \mid f\left(U_{i}\right) \subseteq V_{j}\right\}$ is computably enumerable. Effective $\alpha$-sequences of maps are defined as above.

Fact 2.6. Effective maps are closed under composition. They send effective elements onto effective elements, idem with $\alpha$-sequences for $\alpha<\omega_{1}^{C K}$.

Remark 2.7. If $\left(U_{n}\right)_{n \in \mathbb{N}},\left(V_{n}\right)_{n \in \mathbb{N}}$ are presentations of the topological spaces $P$ and $Q$, then $\left(U_{i} \times V_{j}\right)_{i, j \in \mathbb{N}}$ is a presentation of the product topological space $P \times Q$.

2.4.2. Effective Borel and Hausdorff hierarchies. Fixing a presentation $\left(O_{n}\right)_{n \in \mathbb{N}}$ of the topology of $P$, one defines the effective versions of the Borel and Hausdorff-Kuratowski hierarchies: the classes $\Sigma_{\alpha}^{0}, \Pi_{\alpha}^{0}, \Delta_{\alpha}^{0}, \mathrm{D}_{\alpha}, c o-\mathrm{D}_{\alpha}, \mathrm{D}_{\alpha}\left(\Sigma_{\xi}^{0}\right), c o-\mathrm{D}_{\alpha}\left(\Sigma_{\xi}^{0}\right)$, for $1 \leq \alpha, \xi<\omega_{1}^{C K}$. The class $\Sigma_{1}^{0}$ consists of those open sets which are of the form $\bigcup_{i \in I} O_{i}$ where $I$ is a c.e. (computably enumerable) subset of $\mathbb{N}$. The class $\Sigma_{2}^{0}$ consists of those sets of the form $\bigcup_{n \in \mathbb{N}}\left(\bigcup_{i \in I_{n}} O_{i}\right) \backslash\left(\bigcup_{j \in J_{n}} O_{j}\right)$ where $\left\{(n, i) \mid i \in I_{n}\right\}$ and $\left\{(n, j) \mid i \in J_{n}\right\}$ are c.e. All Borel classes of finite rank are obtained in a similar way. For the infinite ranks, the definition involves a more complex machinery of Borel codes which can be developed in several ways, cf. Moschovakis (1979/2009, Section 3H, 7B), Marker (2002, Section 7), Selivanov (2008, Section 3.2), or Becher and Grigorieff (this volume, Section 5.3-5.5). The effective Hausdorff-Kuratowski classes are obtained similarly. Fact 2.1 has an effective version.

Fact 2.8. If $f: P \rightarrow Q$ is effective then the inverse image of a subset of $Q$ in an effective Borel or Hausdorff-Kuratowski class of $Q$ is in the corresponding effective class of $P$.

\subsubsection{Effective $\omega$-continuous domains. Definition 2.5 extends to domains.}

\section{Definition 2.9.}

1. A presentation of an $\omega$-continuous domain $P$ is an enumeration of some basis of $P$.

2. A presentation $\left(p_{n}\right)_{n \in \mathbb{N}}$ is effective if $\left\{(i, j) \mid p_{i} \ll p_{j}\right\}$ is computably enumerable. An effective $\omega$-continuous domain consists of an $\omega$-continuous domain $P$ together with an effective presentation of $P$.

Fix some presentations $\left(p_{n}\right)_{n \in \mathbb{N}},\left(q_{n}\right)_{n \in \mathbb{N}}$ of the (not necessarily effective) $\omega$-continuous domains $P$ and $Q$.

3. An element $x \in P$ is domain-effective (respectively domain-computable) if the set $\{i \in \mathbb{N} \mid$ $\left.p_{i} \ll x\right\}$ is computably enumerable (respectively computable). For $\alpha<\omega_{1}^{C K}$, domaineffective (respectively domain-computable) $\alpha$-sequences are defined as in Definition 2.5 using the set $\left\{(n, i) \mid p_{i} \ll x_{\rho(n)}\right\}$. Each element of a domain-effective (respectively domain-computable) $\alpha$-sequence is domain-effective (respectively domain-computable).

4. A continuous map $f: P \rightarrow Q$ is domain-effective if $\left\{(i, j) \mid q_{j} \ll f\left(p_{i}\right)\right\}$ is computably enumerable. For $\alpha<\omega_{1}^{C K}$, domain-effective $\alpha$-sequences of maps are defined as above. 
Example 2.10. Consider the sequence $\left(\mathcal{B}_{A}\right)_{A \in \mathcal{P}_{<\omega} \mathbb{N}}$, where $\mathcal{B}_{A}=\{X \in \mathcal{P} \mathbb{N} \mid A \subseteq X\}$. It is an effective presentation of $\mathcal{P} \mathbb{N}$, (called the canonical presentation). An element $X \in \mathcal{P} \mathbb{N}$ is domain-effective (respectively domain-computable) relative to this presentation if and only if it is a computably enumerable (respectively computable) subset of $\mathbb{N}$. A map $f: \mathcal{P} \rightarrow \mathcal{P} \mathbb{N}$ is domain-effective if and only if there exists a computable function $g: \mathcal{P}_{<\omega} \mathbb{N} \rightarrow \mathbb{N}$ such that, for all $X, f(X)=\bigcup_{A \subseteq X, A \in \mathcal{P}_{<\omega} \mathbb{N}} W_{g(A)}$ where $W_{e}$ denotes the computably enumerable subset of $\mathbb{N}$ with code $e$.

Remark 2.11. If $\left(p_{n}\right)_{n \in \mathbb{N}},\left(q_{n}\right)_{n \in \mathbb{N}}$ are presentations of the $\omega$-continuous domains $P$ and $Q$, then $\left(p_{i}, q_{j}\right)_{i, j \in \mathbb{N}}$ is a presentation of the product $\omega$-continuous domain $P \times Q$.

Fact 2.12. Let $\left(p_{n}\right)_{n \in \mathbb{N}},\left(q_{n}\right)_{n \in \mathbb{N}}$ be presentations of the $\omega$-continuous domains $P, Q$. A domain-effective map $f: P \rightarrow Q$ between $\omega$-continuous domains sends any domaineffective element of $P$ to a domain-effective element of $Q$. Idem with domain-effective $\alpha$-sequences for $\alpha<\omega_{1}^{C K}$.

2.4.4. Topological effectiveness versus domain effectiveness. While Definition 2.5 gives the notions of element, sequence and map that are topology-effective, Definition 2.9 does it for the domain-effective notions. Since $x \in \uparrow p_{i} \Leftrightarrow p_{i} \ll x$, for elements and sequences the two definitions of effectivity coincide.

Fact 2.13. Let $\left(p_{n}\right)_{n \in \mathbb{N}}$ be a presentation of the $\omega$-continuous domain $P$. An element $x \in P$ is domain-effective (respectively domain-computable) if and only if it is topology-effective (respectively topology-computable). Idem with $\alpha$-sequences, for $1 \leq \alpha<\omega_{1}^{C K}$.

In the case of $\omega$-algebraic domains, Definitions 2.5 and 2.9, the notion of effectivity coincides not only for elements and sequences but also for space presentations and maps.

Fact 2.14. Let $\left(p_{n}\right)_{n \in \mathbb{N}}$ be a presentation of the $\omega$-algebraic domain $P$ consisting of compact elements. Let $\left(q_{n}\right)_{n \in \mathbb{N}}$ be a presentation of the $\omega$-continuous domain $Q$.

1. The presentation $\left(p_{n}\right)_{n \in \mathbb{N}}$ of the $\omega$-continuous domain is effective if and only if so is the presentation $\left(\uparrow p_{n}\right)_{n \in \mathbb{N}}$ of the Scott topology of $P$.

2. A map $f: P \rightarrow Q$ is domain-effective if and only if it is topology-effective.

Proof. Since $p_{i} \in \uparrow p_{i}$ we have $\uparrow p_{i} \subseteq \uparrow p_{j} \Leftrightarrow p_{j} \ll p_{i}$ and $f\left(\uparrow p_{i}\right) \subseteq \uparrow q_{j} \Leftrightarrow q_{j} \ll f\left(p_{i}\right)$.

Finally, topological effective maps are also domain-effective.

Proposition 2.15. Let $\left(p_{n}\right)_{n \in \mathbb{N}},\left(q_{n}\right)_{n \in \mathbb{N}}$ be presentations of the $\omega$-continuous domains $P, Q$, such that $\left(p_{n}\right)_{n \in \mathbb{N}}$ is domain-effective. Then, a topology-effective map $f: P \rightarrow Q$ is also domain-effective.

Proof. Let $S=\left\{(i, j) \mid q_{j} \ll f\left(p_{i}\right)\right\}$ and $T=\left\{(i, j) \mid f\left(\uparrow p_{i}\right) \subseteq \uparrow q_{j}\right\}$ be the two computably enumerable sets ensuring the domain-effectiveness and the topology-effectiveness of $f$. Inclusion $S \subseteq T$ is straightforward. Though, in general, $S$ and $T$ are distinct, we show that $S=\left\{(i, j) \mid \exists k\left(p_{k} \ll p_{i} \wedge(k, j) \in T\right)\right\}$. In particular, if $T$ is computably enumerable then so is $S$. Implication $p_{k} \ll p_{i} \wedge(k, j) \in T \Rightarrow(i, j) \in S$ is trivial. Conversely, if $(i, j) \in S$ then $q_{j} \ll f\left(p_{i}\right)=\sqcup_{i \in \mathbb{N}} f\left(p_{i_{n}}\right)$ where $\left(p_{i_{n}}\right)_{n \in \mathbb{N}}$ is some increasing chain included in $\downarrow p_{i}$ with 
supremum $p_{i}$. By interpolation (cf. Fact 2.2), let $q_{j} \ll y \ll \sqcup_{i \in \mathbb{N}} f\left(p_{i_{n}}\right)$. By the definition of $\ll$, there is some $n$ such that $y \sqsubseteq f\left(p_{i_{n}}\right)$ hence $q_{j} \ll f\left(p_{i_{n}}\right)$. Thus, letting $k=i_{n}$, we have $p_{k} \ll p_{i}$ and $(k, j) \in S \subseteq T$.

2.4.5. Effective embedding in $\mathcal{P N}$. We give a version of Fact 2.3 that effectivizes the embedding of an $\omega$-continuous domain $P$ in $\mathcal{P}$.

Fact 2.16. Let $\left(p_{n}\right)_{n \in \mathbb{N}}$ be a presentation of the $\omega$-continuous domain $(P, \sqsubseteq)$.

1. An element $x \in P$ is effective (respectively computable) if and only if the set $\varphi(x)=\{n \in$ $\left.\mathbb{N} \mid p_{n} \ll x\right\}$ is effective (respectively computable) relative to the canonical presentation of $\mathcal{P} \mathbb{N}$. Idem with $\alpha$-sequences for $\alpha<\omega_{1}^{C K}$.

2. If the presentation $\left(\uparrow p_{n}\right)_{n \in \mathbb{N}}$ is effective then $\varphi$ is a topology-effective embedding of $(P, \subseteq)$ in $(\mathcal{P} \mathbb{N}, \subseteq)$ (relative to the canonical presentation of $\mathcal{P} \mathbb{N})$. As a consequence, for every effective Borel or Hausdorff-Kuratowski class $\Gamma$,

$$
\{\varphi(Z) \mid Z \in \Gamma(P)\}=\Gamma(\varphi(P))=\{\mathcal{X} \cap \varphi(P) \mid \mathcal{X} \in \Gamma(\mathcal{P N})\}
$$

3. If the presentation $\left(p_{n}\right)_{n \in \mathbb{N}}$ is effective then $\varphi(P)$ is $\Pi_{2}^{0}$ in $\mathcal{P} \mathbb{N}$.

Proof. 2. For $A \in \mathcal{P}_{<\omega} \mathbb{N}, \uparrow A \ll \varphi\left(\uparrow p_{i}\right)$ if and only if $\varphi\left(\uparrow p_{i}\right) \subseteq \mathcal{B}_{A}$ if and only if $\forall n \in A \forall k\left(p_{i} \ll p_{k} \Rightarrow n \in \varphi\left(p_{k}\right)\right)$ if and only if $\forall n \in A \forall k\left(p_{i} \ll p_{k} \Rightarrow p_{n} \ll p_{k}\right)$ if and only if $\forall n \in A\left(\uparrow p_{i} \subseteq \uparrow p_{n}\right)$. The universal quantification is bounded by the finite set $A$, so this last formula gives the computable enumerability of the set $\left\{(A, i) \mid \uparrow A \supseteq \varphi\left(\uparrow p_{i}\right)\right\}$.

3. Observe that in the proof of Fact 2.3 item 3 , the sets $R$ and $\left\{(i, j, k) \mid k \in Z_{i, j}\right\}$ are computably enumerable.

Note 2.17. Item 1 in Fact 2.16 does not need that the presentation $\left(p_{n}\right)_{n \in \mathbb{N}}$ be effective.

\section{Wadge theory and its effectivization}

\subsection{Wadge hardness and completeness}

Definition 3.1 (Wadge 1972). Let $P, Q$ be two topological spaces and $\mathcal{C} \subseteq \mathcal{P}(P)$.

1. $X \subseteq P$ is Wadge reducible to $Y \subseteq Q$, written $X \leq_{W} Y$, if $X=f^{-1}(Y)$ for some continuous $f: P \rightarrow Q$.

2. A set $Y \subseteq Q$ is Wadge hard for $\mathcal{C}$ if every $X \in \mathcal{C}$ is Wadge reducible to $Y$.

3. In case $P=Q$, the set $Y \subseteq Q$ is Wadge complete for $\mathcal{C}$ if it is Wadge hard for $\mathcal{C}$ and it belongs to $\mathcal{C}$.

4. Wadge reducibility, Wadge hardness and Wadge completeness are finite-to-one or one-to-one when the associated reductions are, respectively, finite-to-one or one-to-one.

5. The preordering $\leq_{W}$ on subsets of $P$ induces an equivalence relation. Its equivalence classes are called Wadge degrees.

Definition 3.2. Relative to fixed presentations $\left(U_{n}\right)_{n \in \mathbb{N}}$ and $\left(V_{n}\right)_{n \in \mathbb{N}}$ of the topological spaces $P, Q$, effective Wadge reducibility, hardness and completeness are obtained by requiring that only effective maps be considered in Definition 3.1.

One-to-one and finite-to-one Wadge reducibilities are not equivalent. 
Proposition 3.3. In the Scott domain $\mathcal{P} \mathbb{N}$, Wadge reducibility, finite-to-one and one-to-one reducibilities are not equivalent. Idem with effective reducibilities.

Proof. Let $\mathcal{O}_{1}=\bigcup_{n \in \mathbb{N}} \mathcal{B}_{\{n\}}, \mathcal{O}_{2}=\bigcup_{n>0} \mathcal{B}_{\{n\}}, \mathcal{O}_{3}=\bigcup_{n>0} \mathcal{B}_{\{0, n\}}$. The complements of these families respectively contain one set (namely $\varnothing$ ), two sets (namely $\varnothing$ and $\{0\}$ ), and infinitely many sets (namely $\varnothing,\{0\}$ and all subsets of $\mathcal{P} \mathbb{N} \backslash\{0\}$ ). Thus, there can be no injective reduction of $\mathcal{O}_{2}$ to $\mathcal{O}_{1}$, and no finite-to-one reduction of $\mathcal{O}_{3}$ to both $\mathcal{O}_{1}$ and $\mathcal{O}_{2}$. However, there is a finite-to-one effective reduction of $\mathcal{O}_{2}$ to $\mathcal{O}_{1}$, namely, $f(X)=X \backslash\{0\}$. Also, there is an effective reduction $g$ of $\mathcal{O}_{3}$ to both $\mathcal{O}_{1}$ and $\mathcal{O}_{2}: g(X)=X$, if $0 \in X$ and there is $n>0$ such that $n \in X$; otherwise $g(X)=\varnothing$.

Remark 3.4. Related to the above proposition, cf. Theorem 4.17 and Corollary 4.16.

For zero-dimensional Polish spaces, Wadge theory has beautiful properties.

Theorem 3.5 (Wadge 1972). Let $P, Q$ be zero-dimensional Polish spaces.

1. (Duality theorem). Let $A \subseteq P, B \subseteq Q$ be Borel sets. Then $A \leq_{W} B$ or $B \leq_{W}(P \backslash A)$.

2. (Hardness theorem). For all $\xi<\omega_{1}$, the following conditions are equivalent:

- $A$ is not $\Pi_{\xi}^{0}(P)$.

- $A$ is Wadge hard for $\Sigma_{\xi}^{0}(S)$ for some uncountable zero-dimensional Polish space $S$.

- $A$ is Wadge hard for $\Sigma_{\xi}^{0}(S)$ for every uncountable zero-dimensional Polish space $S$.

Remark 3.6. The zero-dimensional hypothesis is necessary in Theorem 3.5 (cf. Hertling 1996a; 1996b; Ikegami 2010; Schlicht 2011). For instance, consider the closed interval $A=[0,+\infty[$ in the real line. Its complement $\mathbb{R} \backslash A=]-\infty, 0[$ is open. Let $B=\{\alpha \in$ $\left.\mathbb{N}^{\omega}: \forall n \alpha(n) \neq 0\right\}$, so $B$ is not open. Since Wadge reductions are continuous, necessarily $B \Varangle_{W} \mathbb{R} \backslash A$ (a reduction is impossible because the inverse image of an open set by a continuous map must be open) and $A \Varangle_{W} B$ (a reduction is impossible because every continuous map from a connected space to a totally disconnected space is constant).

Wadge duality theorem does not hold in Scott spaces. This contradicts Tang (1981, page 365 line 2) where the theorem is qualified as 'straightforward' for $\mathcal{P} \mathbb{N}$. The failure of the duality theorem leads to the well-known observation that Wadge games, the main tool in the Wadge theory, cannot be used to investigate the Wadge hierarchy on spaces that are not zero-dimensional and Polish.

Proposition 3.7. Wadge duality theorem fails in the Scott domain $\mathcal{P}$.

Proof. Let $\mathcal{X}=\mathcal{B}_{\{0\}}, \mathcal{Y}=\mathcal{P}_{<\omega} \mathbb{N}$. Since $\mathcal{P} \mathbb{N} \backslash \mathcal{X}$ is closed but $\mathcal{Y}$ is not closed, we have $\mathcal{Y} \varliminf_{W} \mathcal{P} \backslash \mathcal{X}$. Let us see that $\mathcal{X} \Varangle_{W} \mathcal{Y}$. Since $2 \mathbb{N}+1 \notin \mathcal{X}$ but $\{0\} \cup 2 \mathbb{N}+1 \in \mathcal{X}$, any reduction $f: \mathcal{P} \mathbb{N} \rightarrow \mathcal{P}$ from $\mathcal{X}$ to $\mathcal{Y}$ should be such that $f(2 \mathbb{N}+1) \notin \mathcal{Y}$ hence infinite and $f(\{0\} \cup 2 \mathbb{N}+1) \in \mathcal{Y}$ hence finite. But this is a contradiction since, being continuous, reductions are increasing with respect to subset inclusion.

Theorem 3.5 does not have an effective version because determinacy is not guaranteed in the effective world. As pointed to us by an anonymous referee, the arguments given by Fokina et al. (2010) yield the following result. 
Proposition 3.8. The ordered structure of inclusion on computable (respectively computably enumerable) subsets of $\mathbb{N}$ can be embedded into the ordered structure of effective Wadge degrees (i.e. degrees relative to computable reductions) of $\Pi_{1}^{0}$ (respectively $\Sigma_{2}^{0}$ ) subsets of the Baire space.

Proof. We follow the arguments in the proof of Theorem 9 in Fokina et al. (2010). Their Theorem 6 establishes that there is a uniform sequence $\left(A_{n}\right)_{n \in \mathbb{N}}$ of nonempty $\Pi_{1}^{0}$ subsets of $\mathbb{N}^{\omega}$ such that, for each $n$, there is no hyperarithmetical function $F: \mathbb{N}^{\omega} \rightarrow \mathbb{N}^{\omega}$ such that $F\left(A_{n}\right) \subseteq \bigcup_{m \neq n} A_{m}$. We also use that the computable sequence $0^{\omega}$ is in no $A_{n}$, stated as a Remark after their Theorem 5.

Case of computable subsets of $\mathbb{N}$. For $f \in \mathbb{N}^{\omega}$ and $k \in \mathbb{N}$, let $k f \in \mathbb{N}^{\omega}$ be such that $(k f)(0)=k$ and $(k f)(x)=f(x+1)$ for all $x \in \mathbb{N}$. For $X \subseteq \mathbb{N}$ computable, let $A_{X}^{*}$ be the $\Pi_{1}^{0}$ set $\bigcup_{n \in X} n A_{n}$. Consider $F: \mathbb{N}^{\omega} \rightarrow \mathbb{N}^{\omega}$ such that, for $f \in \mathbb{N}^{\omega}, F(f)=f$ if $f(0) \in X$ and $F(f)=0^{\omega}$ otherwise. Then $F$ is computable and reduces $A_{X}^{*}$ to $A_{Y}^{*}$ for any $X \subseteq Y \subseteq \mathbb{N}$. Suppose now that $X, Y$ are subsets of $\mathbb{N}, X \nsubseteq Y$, and $F: \mathbb{N}^{\omega} \rightarrow \mathbb{N}^{\omega}$ reduces $A_{X}^{*}$ to $A_{Y}^{*}$. For $g \in \mathbb{N}^{\omega}$, let $g^{-} \in \mathbb{N}^{\omega}$ be obtained by removing the first element of $g$, i.e. $g^{-}(x)=g(x+1)$ for all $x$. Pick some $k \in X \backslash Y$ and let $G: \mathbb{N}^{\omega} \rightarrow \mathbb{N}^{\omega}$ be such that $G(f)=F(k f)^{-}$for all $f \in \mathbb{N}^{\omega}$. Since $k \in X, G\left(A_{k}\right)=F\left(k A_{k}\right)^{-} \subseteq\left(A_{Y}^{*}\right)^{-}=\bigcup_{m \in Y} A_{m}$. Since $k \notin Y$, the assumed property of the $A_{n}$ 's ensures that $G$ hence also the reduction $F$ cannot be hyperarithmetical. Thus, there is no computable reduction of $A_{X}^{*}$ to $A_{Y}^{*}$.

Case of computably enumerable subsets of $\mathbb{N}$. For $X \subseteq \mathbb{N}$ computably enumerable, let $A_{X}^{\dagger}$ be the $\Sigma_{2}^{0}$ set $0^{<\omega} 1 A_{X}^{*}$. Let $\left(n_{i}\right)_{i \in \mathbb{N}}$ be a computable enumeration of $X$ and consider the map $F: \mathbb{N}^{\omega} \rightarrow \mathbb{N}^{\omega}$ such that $F\left(0^{p} 1 n_{i} f\right)=0^{p+i} 1 n_{i} f$ for all $p, i \in \mathbb{N}, f \in \mathbb{N}^{\omega}$, and $F$ takes value $0^{\omega}$ elsewhere. Then $F$ is computable and reduces $A_{X}^{\dagger}$ to $A_{Y}^{\dagger}$ for any $X \subseteq Y$. As before, $A_{X}^{\dagger}$ cannot be effectively reduced to $A_{Y}^{\dagger}$ in case $X \nsubseteq Y$.

\subsection{Universality}

It turns out that universality is related to Wadge completeness, cf. Moschovakis (1979/2009, page 27) or Kechris (1995, page 85, proof of Theorem 14.2).

Definition 3.9. Let $P, Q$ be topological spaces, $\Gamma$ a Borel or Hausdorff-Kuratowski class.

1. A set $U \in \Gamma(P \times Q)$ is $P$-universal for the class $\Gamma(Q)$ if $\Gamma(Q)=\left\{U_{p} \mid p \in P\right\}$ where $U_{p}=\{q \mid(p, q) \in U\}$.

2. A set $S \in \Gamma(Q)$ is strongly $P$-universal for $\Gamma(Q)$ if there exists a continuous map $\Phi: P \times Q \rightarrow Q$ such that $\boldsymbol{\Gamma}(Q)=\left\{\Phi_{p}^{-1}(S) \mid p \in P\right\}$ with $\Phi_{p}(q)=\Phi(p, q)$. If $\Phi$ is one-to-one in its second argument then $S$ is said to be one-to-one strongly $P$-universal.

3. Fix some presentations of $P, Q$. If $\Gamma$ is an effective class, we get corresponding effective notions by requiring equality $\Gamma(Q)=\left\{U_{p} \mid p \in P, p\right.$ effective $\}$ in item 1 and equality $\Gamma(Q)=\left\{\Phi_{p}^{-1}(S) \mid p \in P\right.$, p effective $\}$ with $\Phi$ an effective map in item 2.

\section{Remark 3.10.}

1. If there is some strongly $P$-universal set $S$ for $\Gamma(Q)$ then there is some $P$-universal set $U$ for $\Gamma(Q)$ : let $U=\Phi^{-1}(S)$, for a witness $\Phi$ of the strong $P$-universality of $S$. 
2. Effective strong universality is a variant of the notion of universal partial computable function in Blum's isomorphism theorem (recall that a partial computable $\psi: \mathbb{N} \rightarrow \mathbb{N}$ is universal if there is some computable $f: \mathbb{N}^{2} \rightarrow \mathbb{N}$ such that for all $e, x \in \mathbb{N}$, $\{e\}(x)=\psi(f(e, x))$, cf. Rogers (1967, pages 54, 191)).

Wadge completeness lies between universality and strong universality.

Proposition 3.11. Let $\Gamma$ be a Borel or Hausdorff-Kuratowski class. Let $P, Q$ be topological spaces.

1. If $S$ is (one-to-one) strongly $P$-universal for $\Gamma(Q)$ then $S$ is (one-to-one) Wadge complete for $\Gamma(Q)$. An effective version holds relative to fixed presentations of $P, Q$.

2. Let $C(Q, Q)$ be the set of continuous maps $Q \rightarrow Q$ endowed with any topology at least as fine as the topology of pointwise convergence. If $S$ is Wadge complete for $\Gamma(Q)$ then $U=\{(f, q) \in C(Q, Q) \times Q \mid f(q) \in S\}$ is $C(Q, Q)$-universal for $\boldsymbol{\Gamma}(Q)$. An effective version holds relative to presentations of $Q$ and of a topology on $C(Q, Q)$ finer than that of pointwise convergence.

Proof. 1. Let $X$ be in $\Gamma(Q)$. Since $S$ is strongly universal, there exists $p \in P$ such that $X=\Phi_{p}^{-1}(S)$ where $\Phi_{p}: Q \rightarrow Q$ is the continuous map $q \mapsto \Phi(p, q)$. Notice that if $\Phi$ is injective in the second argument, then $\Phi_{p}$ is injective.

2. The map $a:(f, q) \mapsto f(q)$ is continuous $C(Q, Q) \times Q \rightarrow Q$, so $a^{-1}$ preserves Borel and Hausdorff-Kuratowski classes. Thus, $U=a^{-1}(S) \in \Gamma(C(Q, Q) \times Q)$. Since $S \in \Gamma(Q)$, the set $U_{f}$ is in $\Gamma(Q)$ for all $f \in C(Q, Q)$. Since $S$ is Wadge hard for $\Gamma(Q)$, every $X \in \boldsymbol{\Gamma}(Q)$ is of the form $X=f^{-1}(S)$ for some $f \in C(Q, Q)$. Hence, $f^{-1}(S)=U_{f}$. If $Q$ is not a countable set, the pointwise topology on $C(Q, Q)$ has no countable basis. For the effective version we need a finer topology: If $\left(V_{i}\right)_{i \in \mathbb{N}}$ is a presentation of $Q$, a convenient topology on $C(Q, Q)$ has a presentation $\left(\mathcal{V}_{A}\right)_{A \in \mathcal{P}_{<\omega} \mathbb{N}^{2}}$ where $\mathcal{V}_{A}=\left\{f \in C(Q, Q) \mid \forall(i, j) \in A f\left(U_{i}\right) \subseteq U_{j}\right\}$.

Wadge completeness coincides with strong universality when this last condition is not void.

Fact 3.12. If there is a strongly $P$-universal set for $\Gamma(Q)$, then every Wadge complete set for $\Gamma(Q)$ is strongly $P$-universal for $\Gamma(Q)$. One-to-one and effective versions also hold.

Proof. Suppose $U$ is a strongly $P$-universal set for $\Gamma(Q)$ and $V$ is a Wadge complete set for $\Gamma(Q)$. Any continuous reduction from $V$ to $U$ composed with a witness of the strongly $P$-universality of $U$ yields a witness of the strongly $P$-universality of $V$.

There are also cases in which universality grants Wadge completeness.

Fact 3.13. Suppose $h: P \times Q \rightarrow Q$ is a homeomorphism. If $U$ is $P$-universal for $\Gamma(Q)$ then $h(U)$ is one-to-one Wadge complete for $\Gamma(Q)$.

By classical arguments (Kechris, 1995), Wadge hardness and $P$-universality can be lifted with set-theoretical operations of complements, (countable) unions, intersections and differences. When $P, P^{2}$ and $P^{\omega}$ (endowed with the product topologies) are all homeomorphic (for example $N^{\omega}, 2^{\omega}$ or $\mathcal{P} \mathbb{N}$ ), one can lift Wadge completeness (respectively, 
$P$-universality) for a class $\mathbf{D}_{\alpha}\left(\boldsymbol{\Sigma}_{\xi}(Q)\right)$ to that for a class $\mathbf{D}_{\beta}\left(\boldsymbol{\Sigma}_{\mu}(Q)\right)$ as long as either $\xi<\mu$ or else $\xi=\mu$ and $\alpha$ is less or equal to $\beta$.

\subsection{Known outside the Baire space}

The following simple result shows that $\mathcal{P N}$ is for $\omega$-continuous domains what the Baire space $\mathbb{N}^{\omega}$ is for Polish spaces. It can be seen as an extension of Facts 2.3 and 2.16.

Proposition 3.14. For every Polish space (respectively $\omega$-continuous domain) $Q$ there is a $\mathbb{N}^{\omega}$-universal (respectively $\mathcal{P} \mathbb{N}$-universal) set for $\Sigma_{1}^{0}(Q)$. Given presentations of the topological spaces $\mathbb{N}^{\omega}$ and $Q$ (respectively the $\omega$-continuous domains $\mathcal{P}$ and $Q$ ), this universal set is in the effective class relative to the associated presentation of $\mathbb{N}^{\omega} \times Q$ (respectively $\mathcal{P} \mathbb{N} \times Q$ ), cf. Remarks 2.7 and 2.11 .

Proof. We argue for the case of $\omega$-continuous domains. Let $\left(b_{i}\right)_{i \in \mathbb{N}}$ be a presentation of $Q$. Let $U=\left\{(X, x) \in \mathcal{P} \mathbb{N} \times Q \mid \exists i \in X b_{i} \ll x\right\}$. Observe that $U \in \Sigma_{1}^{0}(\mathcal{P} \mathbb{N} \times Q)$ since $U=\bigcup_{i \in \mathbb{N}} \mathcal{B}_{\{i\}} \times \uparrow b_{i}$. Clearly, $U$ is in the effective class $\Sigma_{1}^{0}(\mathcal{P} \mathbb{N} \times Q)$. Also, $U$ is $\mathcal{P} \mathbb{N}$-universal for $\Sigma_{1}^{0}(Q)$ since, for every $I \subseteq \mathbb{N}$, the open set $O=\bigcup_{i \in I} \uparrow b_{i}$, of $Q$ is equal to $U_{I}$.

Applying Fact 3.13 and Proposition 3.14, one obtains the existence of Wadge complete sets for domains such as $\mathcal{P} \mathbb{N}$, a result proved in Selivanov (2005b).

Corollary 3.15 (Selivanov 2005b). If an $\omega$-continuous domain $Q$ is homeomorphic to $\mathcal{P} \times Q$ then it admits some one-to-one Wadge complete set for each Borel or HausdorffKuratowski class.

Tang (1979) proved that a family $\mathcal{A} \in \Delta_{2}^{0}(\mathcal{P N})$ is in $\bigcup_{n<\omega} \mathbf{D}_{n}(\mathcal{P} \mathbb{N})$, if and only if there is a finite bound on the length of $\mathcal{A}$-alternating monotone chains. Of course, for finite chains there is no distinction between increasing or decreasing chains. Using alternating trees, Selivanov $(2005 b$; 2006) extended and refined this result for $\omega$-algebraic domains. A slight variation of the proof goes through with $\omega$-continuous domains, cf. Selivanov (2008), Becher and Grigorieff (this volume).

Proposition 3.16 (Selivanov 2005b, Proposition 6.4 $i$ ). Let $P$ be an $\omega$-continuous domain and $n \in \mathbb{N}$. Every set in $\Delta_{2}^{0}(P)$ but not in $c o-\mathbf{D}_{n}(P)$ is Wadge hard for $\mathbf{D}_{n}(P)$.

Polish spaces and $\omega$-continuous domains have been put in an elegant unifying framework: the quasi-Polish spaces (de Brecht 2011). Up to homeomorphism, quasi-Polish spaces coincide with the $\Pi_{2}^{0}$ subspaces of $\mathcal{P}$. Wadge hardness results transfer from $\mathcal{P} \mathbb{N}$ to quasi-Polish spaces as follows.

Proposition 3.17. Let $\boldsymbol{\Gamma}$ is $\boldsymbol{\Sigma}_{\alpha}^{0}(\mathcal{P} \mathbb{N})$ with $\alpha \geq 3$ or $\Pi_{\alpha}^{0}(\mathcal{P N})$ with $\alpha \geq 2$. Let $P$ be a topological space. Any set $H \subseteq P$ that is Wadge hard for $\Gamma(\mathcal{P N})$ is also Wadge hard for $\Gamma(Q)$ for every quasi-Polish space $Q$.

Proof. Since every quasi-Polish space is homeomorphic to a $\Pi_{2}^{0}$ subspace of $\mathcal{P}$, we can suppose $Q$ is a $\Pi_{2}^{0}$ subspace of $\mathcal{P} \mathbb{N}$. The hypothesis on $\Gamma$ ensures that $Q$ is in $\Gamma(\mathcal{P} \mathbb{N})$. 
If $A \in \boldsymbol{\Gamma}(Q)$, then $A=A^{\prime} \cap Q$ for some $A^{\prime} \in \Gamma(\mathcal{P N})$ hence $A \in \Gamma(\mathcal{P} \mathbb{N})$. The restriction to $Q$ of any continuous reduction of $A^{\prime}$ to $H$ is a reduction of $A$ to $H$.

\section{Wadge hardness and alternating decreasing chains}

\subsection{Alternating chains}

Recall that we write $\alpha \sim \beta$ to indicate that $\alpha$ and $\beta$ have the same parity.

Definition 4.1. Let $(P$, ㄷ) be a dcpo, $A$ a subset of $P$ and $\alpha$ an ordinal.

1. An $(\alpha+1)$-sequence $\left(x_{\beta}\right)_{\beta \leq \alpha}$ of elements of $P$ is an A-alternating decreasing chain if

- $x_{\beta} \sqsupset x_{\delta}$ for all $\beta<\delta \leq \alpha$, and

- $x_{\beta} \in A$ if and only if $\beta \psi \alpha$ (thus, $x_{\alpha} \notin A$ ).

2. An $A$-special $(\alpha+1)$-chain is an $A$-alternating decreasing chain $\left(x_{\beta}\right)_{\beta \leq \alpha}$ such that

$-\left(x_{\beta} \sqsupseteq x \sqsupset x_{\beta+1}\right) \Rightarrow(x \in A \Leftrightarrow \beta \psi \alpha)$, and

$-\left(x_{\alpha} \sqsupseteq x\right) \Rightarrow(x \notin A)$.

Note 4.2. If $\left(x_{\beta}\right)_{\beta \leq \alpha}$ is $A$-alternating and $\gamma<\alpha$, then $\left(x_{\beta}\right)_{\beta \leq \gamma}$ is $A$-alternating if and only if $\gamma \sim \alpha$.

Remark 4.3. As proved by Selivanov (2005b; 2008), when $\alpha$ is infinite and $A$ is $\Delta_{2}^{0} A$ alternating increasing chains do not exist. In fact, if $A$ is in the difference of two open sets and the supremum of an increasing chain $\left(x_{n}\right)_{n \in \mathbb{N}}$ is in $A$ then the $x_{n}$ 's are in $A$ for all $n$ large enough. By considering countable unions, it follows that the same is true if $A$ is $\Sigma_{2}^{0}$. This property forbids $A$-alternation of increasing infinite chains if $A$ is $\Delta_{2}^{0}$.

\subsection{Wadge hardness and alternating decreasing $(\alpha+1)$-chains}

If $A$ is in $\Delta_{2}^{0}$ then long $A$-alternating decreasing infinite chains do exist in $\mathcal{P}$.

Proposition 4.4. Let $\alpha \geq 1$ be a countable ordinal.

1. There exists a family $\mathcal{A} \in \mathbf{D}_{\alpha}(\mathcal{P N})$ and an $\mathcal{A}$-special chain $\left(X_{\beta}\right)_{\beta \leq \alpha}$ such that $X_{\alpha}$ and $X_{\beta} \backslash X_{\beta+1}$, for all $\beta<\alpha$, are infinite subsets of $\mathbb{N}$.

2. If $\alpha<\omega_{1}^{C K}$ then $\mathcal{A}$ can be taken in the effective class $\mathrm{D}_{\alpha}(\mathcal{P} \mathbb{N})$ and the chain can be taken computable (cf. Definition 2.9).

Proof. Let $\beta \mapsto a_{\beta}$ be a bijection $\alpha \rightarrow 3 \mathbb{N}$. Let $\left(A_{\beta}\right)_{\beta \leq \alpha}$ be a family of pairwise disjoint infinite subsets of $3 \mathbb{N}+1$. For $\beta \leq \alpha$, let $X_{\beta}=(3 \mathbb{N}+2) \cup \cup_{\beta \leq \delta<\alpha}\left(\left\{a_{\delta}\right\} \cup A_{\delta}\right)$. Then $X_{\beta} \backslash X_{\beta+1}=\left\{a_{\beta}\right\} \cup A_{\beta}$ and $X_{\alpha}=3 \mathbb{N}+2$ are infinite sets. For $\beta<\alpha$, define an open family $U_{\beta}=\left\{Z \mid \exists \gamma \leq \beta \quad a_{\gamma} \in Z\right\}$. With these open families, define a family $\mathcal{A}=D_{\alpha}\left(\left(U_{\beta}\right)_{\beta<\alpha}\right)=$ $\cup_{\beta<\alpha, \beta, \alpha} U_{\beta} \backslash \bigcup_{\gamma<\beta} U_{\gamma}$ in $\mathbf{D}_{\alpha}(\mathcal{P} \mathbb{N})$. Observe that a set $X$ is in $\mathcal{A}$, if and only if it meets $\left\{a_{\beta} \mid \beta<\alpha\right\}$ and the least $\beta<\alpha$ such that $a_{\beta} \in X$ has parity different from that of $\alpha$. In particular, if $X_{\beta} \supseteq X \supset X_{\beta+1}$ then $X \in \mathcal{A}$ if and only if $\beta \psi \alpha$. Thus $\left(X_{\beta}\right)_{\beta \leq \alpha}$ is an $A$-special chain. Effectivization is straightforward. 
In general, in continuous domains, long decreasing chains may not exist. For instance in the $\omega$-algebraic domain $\left(\mathbf{2}^{\leq \omega}\right.$, 드) of finite and infinite binary words with the prefix ordering, every decreasing chain is finite. However, in case a long decreasing chain exists then it can always be viewed as an $A$-alternating chain for some $A \in \Delta_{2}^{0}$.

Proposition 4.5. Let $P$ be a continuous domain and $\alpha<\omega_{1}$ be an ordinal.

1. Every strictly decreasing chain $\left(x_{\beta}\right)_{\beta \leq \alpha}$ in $P$ is $A$-special for some $A \in \mathbf{D}_{\alpha}(P)$.

2. Suppose $P$ is $\omega$-continuous and fix some presentation of $P$. If $\alpha<\omega_{1}^{C K}$ and the chain is computable then we can take $A \in \mathrm{D}_{\alpha}(P)$.

Proof. Let $B$ be a basis of $P$. In the vein of Fact 2.3, we use the isomorphism $\psi$ between $(P, ㄷ)$ and a subspace of $(\mathcal{P}(B), \subseteq)$ such that $\psi(x)=B \cap \downarrow x=\{b \in B \mid b \ll x\}$. Thus, $\left(\psi\left(x_{\beta}\right)\right)_{\beta \leq \alpha}$ is a strictly decreasing chain. Let $U_{\beta}=\bigcup\left\{\uparrow b \mid b \in \psi\left(x_{0}\right) \backslash \psi\left(x_{\beta+1}\right)\right\}$ for $\beta<\alpha$. The $U_{\beta}$ 's are strictly increasing open subsets of $P$. Let $A=D_{\alpha}\left(\left(U_{\beta}\right)_{\beta<\alpha}\right)$. The set $A$ is in $\mathbf{D}_{\alpha}(P)$. Suppose $\beta<\alpha$ and $x_{\beta} \sqsupseteq x \sqsupset x_{\beta+1}$. Then $\psi\left(x_{\beta}\right) \supseteq \psi(x) \supset \psi\left(x_{\beta+1}\right)$, so that $x \in U_{\delta}$ if and only if $\psi(x)$ meets $\psi\left(x_{0}\right) \backslash \psi\left(x_{\delta+1}\right)$ if and only if $\delta \geq \beta$. In particular, $x \in U_{\beta} \backslash \bigcup_{\gamma<\beta}$. Thus, $x \in A$ if and only if $\beta \neq \alpha$ (hence, $\beta \sim \alpha+1$ ). Finally, suppose $x_{\alpha} \sqsupseteq x$. Then $\psi\left(x_{\alpha}\right) \supseteq \psi(x)$ and $x$ is in no $U_{\beta}$, hence $x \notin A$. For the effective version, using a presentation $\left(b_{i}\right)_{i \in \mathbb{N}}$, replace $\psi$ with $\varphi$ of Facts 2.3 and 2.16 such that $\varphi(x)=\left\{i \in \mathbb{N} \mid b_{i} \ll x\right\}$. The computability of the chain $\left(x_{\beta}\right)_{\beta \leq \alpha}$ ensures that of $\left(\varphi\left(x_{0}\right) \backslash \varphi\left(x_{\beta+1}\right)\right)_{\beta \leq \alpha}$.

Note 4.6. An effective chain is not enough for the above proof: being differences of computably enumerable sets, the sets $\varphi\left(x_{0}\right) \backslash \varphi\left(x_{\beta+1}\right)$ might not be computably enumerable.

Remark 4.7. The proof of Proposition 4.5 amounts to a proof for the case $P=\mathcal{P}(\kappa)$, where $\kappa$ is cardinal, and a transfer to all continuous domains having a basis of cardinality $\leq \kappa$ via an obvious extension of Fact 2.3. Idem for effectivization with $\kappa=\omega$ and Fact 2.16.

We present now one of the main theorems of the paper. For the effective part of the result we use the following convention: effective reductions $P \rightarrow Q$ are relative to the presentations $\left(\uparrow p_{n}\right)_{n \in \mathbb{N}}$ and $\left(\uparrow q_{n}\right)_{n \in \mathbb{N}}$ of the Scott topologies on $P, Q$ associated to presentations $\left(p_{n}\right)_{n \in \mathbb{N}}$ and $\left(q_{n}\right)_{n \in \mathbb{N}}$ of the $\omega$-continuous domains $P, Q$.

Theorem 4.8. Let $Q$ be a continuous domain, $H \subseteq Q$ and $\alpha<\omega_{1}$ be an ordinal.

1. The following conditions are equivalent.

(i) $H$ is Wadge hard for $\mathbf{D}_{\alpha}(P)$ for every continuous domain $P$.

(ii) $H$ is Wadge hard for $\mathbf{D}_{\alpha}(\mathcal{P N})$.

(iii) $H$ is Wadge hard for $\mathbf{D}_{\alpha}(P)$ for some continuous domain $P$ admitting a strictly decreasing chain of length $\alpha+1$.

(iv) There exists a decreasing $H$-alternating chain in $Q$ of length $\alpha+1$.

2. Suppose $Q$ is an $\omega$-continuous domain and fix some presentation $\left(q_{n}\right)_{n \in \mathbb{N}}$ of $Q$. If $\alpha<\omega_{1}^{C K}$ the following conditions are equivalent.

$\left(i_{e}\right) H$ is Wadge hard for $\mathbf{D}_{\alpha}(P)$ and effectively Wadge hard for $\mathrm{D}_{\alpha}(P)$ for every effective $\omega$-continuous domain $P$ (cf. Definition 2.9). 
(ii $) H$ is effectively Wadge hard for $\mathrm{D}_{\alpha}(\mathcal{P N})$.

$\left(\right.$ iii $\left._{e}\right) H$ is effectively Wadge hard for $\mathrm{D}_{\alpha}(P)$ for some effective $\omega$-algebraic domain $P$ admitting a computable strictly decreasing chain of length $\alpha+1$.

$\left(i v_{e}\right)$ There exists an effective decreasing $H$-alternating chain in $Q$ of length $\alpha+1$.

Proof. $(i) \Rightarrow$ (ii). Particularize item (i) with $P=\mathcal{P}$.

(ii) $\Rightarrow$ (iii). Let $P=\mathcal{P} \mathbb{N}$ and use Proposition 4.4.

(iii) $\Rightarrow($ iv). By Proposition 4.5, the strictly decreasing chain in $P$ of length $\alpha+1$ given by (iii) is $A$-alternating for some $A \in \mathbf{D}_{\alpha}(P)$. A continuous reduction of $A$ to $H$ maps this $A$-alternating chain onto a decreasing $H$-alternating chain in $Q$.

(iv) $\Rightarrow(i)$. Let $\left(y_{\beta}\right)_{\beta \leq \alpha}$ be a decreasing $H$-alternating chain of length $\alpha+1$. Suppose $X$ is a set in $\mathbf{D}_{\alpha}(P)$. Then $X=\cup_{\beta<\alpha, \beta \psi_{\alpha}} V_{\beta} \backslash \bigcup_{\gamma<\beta} V_{\gamma}$ for some increasing chain $\left(V_{\beta}\right)_{\beta \leq \alpha}$ of open sets. Define $\tau: P \rightarrow \alpha+1$ and $f: P \rightarrow Q$ as follows: for $z \in P$,

$$
\begin{aligned}
\tau(z) & = \begin{cases}\text { least } \beta<\alpha \text { such that } z \in V_{\beta} & \text { if } z \in \cup_{\beta<\alpha} V_{\beta} \\
\alpha & \text { otherwise. }\end{cases} \\
f(z) & =y_{\tau(z)} .
\end{aligned}
$$

Since the $V_{\beta}$ 's are increasing and are open hence are upsets in $P, \tau$ is decreasing and $f$ is increasing. Letting $B$ be a basis of $P$, we have $V_{\beta}=\bigcup_{c \in I_{\beta}} \uparrow c$ for some $I_{\beta} \subseteq B$. Thus,

$$
\begin{aligned}
z \in V_{\beta} & \Leftrightarrow \exists c \in I_{\beta} \quad c \ll z \\
& \Leftrightarrow \exists c \in I_{\beta} \quad \exists b \in B \quad c \ll b \ll z \\
& \Leftrightarrow \exists b \in B\left(b \ll z \wedge b \in V_{\beta}\right) \\
\tau(z) & =\min _{\{}\{(b) \mid b \in B, b \ll z\} \\
f(z) & =\max _{\sqsubseteq}\{f(b) \mid b \in B, b \ll z\} .
\end{aligned}
$$

(by the interpolation property)

The last equality shows that $f$ is continuous. Finally, observe that $z \in X$ if and only if $\tau(z)<\alpha$ and $\tau(z) \psi \alpha$ if and only if $f(z)=y_{\tau(z)} \in H$. Thus, $f$ reduces $X$ to $H$.

$\left(i_{e}\right) \Rightarrow\left(i i_{e}\right) \Rightarrow\left(i i_{e}\right)$. Idem as $(i) \Rightarrow(i i) \Rightarrow(i i i)$.

$\left(\right.$ iii $\left._{e}\right) \Rightarrow\left(i_{e}\right)$. By Proposition 4.5 , the chain in $P$ of length $\alpha+1$ given by (iii) is $A$-alternating for some $A \in \mathrm{D}_{\alpha}(P)$. Observe that an effective reduction from $A$ to $H$ maps this computable chain onto an effective decreasing $H$-alternating chain.

$\left(i v_{e}\right) \Rightarrow\left(i_{e}\right)$. Suppose $X \in \mathrm{D}_{\alpha}(P)$. Keeping the notation as in the proof of $(i v) \Rightarrow(i)$, we first show that the reduction $f: P \rightarrow Q$ of $X$ to $H$ is domain-effective. Let $\left(p_{i}\right)_{i \in \mathbb{N}}$ and $\left(q_{\ell}\right)_{\ell \in \mathbb{N}}$ be presentations of $P$ and $Q$ such that $\left(p_{i}\right)_{i \in \mathbb{N}}$ is effective, i.e. $\left\{(i, j) \mid p_{i} \ll p_{j}\right\}$ is computably enumerable. Since $\alpha<\omega_{1}^{C K}$ and the chain $\left(y_{\beta}\right)_{\beta \leq \alpha}$ is effective, there exists an initial segment $S$ of $\mathbb{N}$, a set $R \subset S^{2}$ and a map $\rho: S \rightarrow \alpha+1$ such that

- $\rho$ is an isomorphism (necessarily unique) between $(S, R)$ and $(\alpha+1, \leq)$,

- the relation $\left\{(n, \ell) \mid q_{\ell} \ll y_{\rho(n)}\right\}$ is computably enumerable.

Since $X \in \mathrm{D}_{\alpha}(P)$, we can suppose that $\left\{(n, j) \mid p_{j} \in I_{\rho(n)}\right\}$ is a computably enumerable set. Let $a \in S$ be such that $\alpha=\rho(a)$. For $\ell, i \in \mathbb{N}$, 


$$
\begin{aligned}
q_{\ell} \ll f\left(p_{i}\right) & \Leftrightarrow q_{\ell} \ll y_{\tau\left(p_{i}\right)} \\
& \Leftrightarrow q_{\ell} \ll \max \left(\left\{y_{\beta} \mid \tau\left(p_{i}\right) \leq \beta \leq \alpha\right\}\right) \\
& \Leftrightarrow q_{\ell} \ll y_{\alpha} \text { or } \exists \beta<\alpha\left(q_{\ell} \ll y_{\beta} \text { and } \beta \geq \tau\left(p_{i}\right)\right) \\
& \Leftrightarrow q_{\ell} \ll y_{\alpha} \text { or } \exists \beta<\alpha\left(q_{\ell} \ll y_{\beta} \text { and } p_{i} \in V_{\beta}\right) \\
& \Leftrightarrow q_{\ell} \ll y_{\alpha} \text { or } \exists \beta<\alpha\left(q_{\ell} \ll y_{\beta} \text { and } \exists p_{j} \in I_{\beta} p_{j} \ll p_{i}\right) \\
& \Leftrightarrow q_{\ell} \ll y_{\rho(a)} \text { or } \exists n \neq a \exists j\left(q_{\ell} \ll y_{\rho(n)} \text { and } p_{j} \in I_{\rho(n)} \text { and } p_{j} \ll p_{i}\right) .
\end{aligned}
$$

Thus, the set $\left\{(i, \ell) \mid q_{\ell} \ll f\left(p_{i}\right)\right\}$ is obtained via conjunction, disjunction and projection of computably enumerable sets. As such, it is computably enumerable. This proves $f$ is a domain-effective map.

If $P$ is $\omega$-algebraic and the $p_{n}$ 's are compact elements then Fact 2.14 ensures that the reduction $f$ is also topology-effective. In the general case where $P$ is only $\omega$-continuous, we argue as follows. Consider the map $\varphi: P \rightarrow \mathcal{P N}$ such that $\varphi(x)=\left\{n \in \mathbb{N} \mid p_{n} \ll x\right\}$. Since the presentation $\left(p_{n}\right)_{n \in \mathbb{N}}$ of $P$ is effective, Fact 2.16 ensures that $\varphi$ is a topological embedding which is topology-effective (relatively to the canonical presentation of $\mathcal{P} \mathbb{N}$ ) and that $\varphi(X)=\mathcal{Y} \cap \varphi(P)$ for some $\mathcal{Y} \in \mathrm{D}_{\alpha}(\mathcal{P} \mathbb{N})$. Since $\mathcal{P} \mathbb{N}$ is an effective $\omega$-algebraic domain, the above argument applied to the subset $\mathcal{Y}$ of $\mathcal{P} \mathbb{N}$, yields a reduction $g: \mathcal{P} \rightarrow Q$ of $\mathcal{Y}$ to $H$ which is topology-effective. The composition $g \circ \varphi: P \rightarrow Q$ is then a reduction of $X$ to $H$ which is also topology-effective.

Remark 4.9. Letting $\alpha=1$, the characterization of hardness for $\Sigma_{1}^{0}(\mathcal{P} \mathbb{N})$ given by Theorem 4.8 is in the vein of the work done in Becher and Grigorieff (2009).

The next Corollary was known for the domain $\mathcal{P} \mathbb{N}$ (Selivanov 2005b), cf. Corollary 3.15.

Corollary 4.10. Let $Q$ be a continuous domain and $\alpha<\omega_{1}$ be a countable ordinal.

1. If $Q$ admits some strictly decreasing chain of length $\alpha+1$ then there exists a Wadge complete set for $\mathbf{D}_{\alpha}(Q)$. In particular, this applies if $Q=\mathcal{P} \mathbb{N}$.

2. Suppose $Q$ is an effective $\omega$-continuous domain. If $Q$ admits some computable strictly decreasing chain of length $\alpha+1$ then there exists a Wadge complete set for $\mathbf{D}_{\alpha}(Q)$ which is also effectively Wadge complete for $\mathrm{D}_{\alpha}(Q)$. In particular, this applies if $Q=\mathcal{P} \mathbb{N}$.

Proof. By Proposition 4.5, the assumed strictly decreasing chain of length $\alpha+1$ is $H$-special, hence $H$-alternating for some $H \in \mathbf{D}_{\alpha}(Q)$. Thus item (iv) of Theorem 4.8 is satisfied; then, item (i) of Theorem 4.8 also holds, hence $H$ is as desired. The possible instantiation $Q=\mathcal{P} \mathbb{N}$ comes from Proposition 4.4. Effectivization is straightforward.

\subsection{One-to-one Wadge completeness and alternating decreasing chains}

Universal properties as those in part $1(i)$ of Theorem 4.8 cannot hold with one-to-one Wadge reductions $f: P \rightarrow Q$ since such reductions can only exist if $Q$ has cardinality at least that of $P$. Therefore, we shall restrict to $\omega$-continuous domains. The version of Theorem 4.8 for one-to-one hardness relies on the following notion.

Definition 4.11. Let $(P, \sqsubseteq)$ be an ordered set and $\alpha$ an ordinal.

1. A strictly decreasing chain $\left(x_{\beta}\right)_{\beta \leq \alpha}$ is scattered if there exist one-to-one continuous maps $\theta_{\beta}: \mathcal{P} \mathbb{N} \rightarrow P$, for $\beta \leq \alpha$, such that

$$
\theta_{\alpha}(\mathcal{P N}) \subseteq\left\{x \mid x_{\alpha} \sqsupseteq x\right\} \text {, and } \theta_{\beta}(\mathcal{P} \mathbb{N}) \subseteq\left\{x \mid x_{\beta} \sqsupseteq x \sqsupset x_{\beta+1}\right\} \text { for } \beta<\alpha .
$$


2. Let $A \subseteq P$. A strictly decreasing chain $\left(x_{\beta}\right)_{\beta \leq \alpha}$ is $A$-scattered if it is scattered for one-to-one continuous maps $\theta_{\beta}: \mathcal{P} \mathbb{N} \rightarrow P$, for $\beta \leq \alpha$, such that

$$
\theta_{\beta}(\mathcal{P N}) \subseteq A \text { if } \beta \psi \alpha \text {, and } \theta_{\beta}(\mathcal{P N}) \subseteq P \backslash A \text { if } \beta \sim \alpha
$$

3. Suppose $(P, \sqsubseteq)$ is an $\omega$-continuous domain. Fix some presentation of $P$. If $\alpha<\omega_{1}^{C K}$, a scattered chain $\left(x_{\beta}\right)_{\beta \leq \alpha}$ is effective if it is an effective $(\alpha+1)$-sequence of elements of $P$ and $\left(\theta_{\beta}\right)_{\beta \leq \alpha}$ is an effective $(\alpha+1)$-sequence of maps $\mathcal{P} \mathbb{N} \rightarrow P$.

Proposition 4.12. Let $\left(X_{\beta}\right)_{\beta \leq \alpha}$ be a strictly decreasing sequence of subsets of $\mathbb{N}$, of length $(\alpha+1)$.

1. The following conditions are equivalent.

(a) The chain $\left(X_{\beta}\right)_{\beta \leq \alpha}$ is scattered.

(b) $X_{\alpha}$ and each of $X_{\beta} \backslash X_{\beta+1}$, for $\beta<\alpha$, is an infinite set.

2. Suppose $\left(X_{\beta}\right)_{\beta \leq \alpha}$ is $\mathcal{A}$-special for some $\mathcal{A} \subseteq \mathcal{P} \mathbb{N}$. Then, a third equivalent condition is (c) $\left(X_{\beta}\right)_{\beta \leq \alpha}$ is $\mathcal{A}$-scattered.

Proof. $(a) \Rightarrow(b)$. Suppose the chain is scattered. Then, with the notation of Definition 4.11, for $\beta<\alpha,\left(\theta_{\beta}(\{0, \ldots, n\})\right)_{n \in \mathbb{N}}$ is a strictly increasing sequence of sets in $\left\{X \mid X_{\beta} \supseteq X \supset X_{\beta+1}\right\}$. Thus, $X_{\beta} \backslash X_{\beta+1}$ is infinite. Similarly, $X_{\alpha}$ is infinite.

$(b) \Rightarrow(a)$. Consider bijective maps $\mu_{\alpha}: \mathbb{N} \rightarrow X_{\alpha}$ and $\mu_{\beta}: \mathbb{N} \rightarrow X_{\beta} \backslash X_{\beta+1}$, for $\beta<\alpha$. For $Z \in \mathcal{P} \mathbb{N}$, let $\theta_{\alpha}(Z)=\mu_{\alpha}(Z)$ and, for $\beta<\alpha, \theta_{\beta}(Z)=X_{\beta+1} \cup \mu_{\beta}(Z)$. The condition of Definition 4.11 item 1 is true, hence the chain is scattered.

In case the chain is $\mathcal{A}$-special, $(b) \Rightarrow(c)$ and $(c) \Rightarrow(a)$ are straightforward.

Corollary 4.13. Let $\alpha<\omega_{1}$ be an ordinal. There exists $\mathcal{A} \in \mathbf{D}_{\alpha}(\mathcal{P} \mathbb{N})$ and an $\mathcal{A}$-scattered chain of length $\alpha+1$. If $\alpha<\omega_{1}^{C K}$ then we can take $\mathcal{A} \in \mathrm{D}_{\alpha}(\mathcal{P} \mathbb{N})$ and get a computable $\mathcal{A}$-scattered chain of length $\alpha+1$.

Proof. Use Propositions 4.4 and 4.12 .

To characterize effective Wadge one-to-one hardness we use the same convention as in Theorem 4.8: effective reductions $P \rightarrow Q$ are relative to the presentations $\left(\uparrow p_{n}\right)_{n \in \mathbb{N}}$ and $\left(\uparrow q_{n}\right)_{n \in \mathbb{N}}$ of the Scott topologies on $P, Q$ for presentations $\left(p_{n}\right)_{n \in \mathbb{N}}$ and $\left(q_{n}\right)_{n \in \mathbb{N}}$ of the $\omega$-continuous domains $P, Q$.

Theorem 4.14. Let $Q$ be an $\omega$-continuous domain, $H \subseteq Q$ and $\alpha<\omega_{1}$ be an ordinal.

1. The following conditions are equivalent.

(i) $H$ is one-to-one Wadge hard for $\mathbf{D}_{\alpha}(P)$ for every $\omega$-continuous domain $P$.

(ii) $H$ is one-to-one Wadge hard for $\mathbf{D}_{\alpha}(\mathcal{P} \mathbb{N})$.

(iii) $H$ is one-to-one Wadge hard for $\mathbf{D}_{\alpha}(P)$ for some continuous domain $P$ admitting a scattered chain of length $\alpha+1$.

(iv) There exists an $H$-scattered chain in $Q$ of length $\alpha+1$.

2. Fix some presentation $\left(q_{n}\right)_{n \in \mathbb{N}}$ of $Q$. If $\alpha<\omega_{1}^{C K}$ the following conditions are equivalent.

$\left(i_{e}\right) H$ is one-to-one Wadge hard for $\mathbf{D}_{\alpha}(P)$ and effectively one-to-one Wadge hard for $\mathrm{D}_{\alpha}(P)$ for every effective $\omega$-continuous domain $P$ (cf. Definition 2.9). 
(iie) $H$ is effectively one-to-one Wadge hard for $\mathrm{D}_{\alpha}(\mathcal{P} \mathbb{N})$.

$\left(\right.$ iii $\left._{e}\right) H$ is effectively one-to-one Wadge hard for $\mathrm{D}_{\alpha}(P)$ for some effective $\omega$-algebraic domain $P$ admitting a computable scattered chain of length $\alpha+1$.

$\left(i v_{e}\right)$ There exists an effective $H$-scattered chain in $Q$ of length $\alpha+1$.

Proof. $($ i $) \Rightarrow$ (ii). Particularize item $(i)$ with $P=\mathcal{P}$.

(ii) $\Rightarrow$ (iii). Let $P=\mathcal{P} \mathbb{N}$ and use Corollary 4.13 .

(iii) $\Rightarrow($ iv $)$. By Proposition 4.5, the strictly decreasing chain in $P$ of length $\alpha+1$ given by $(i)$ is $A$-special for some $A \in \mathbf{D}_{\alpha}(P)$. Being scattered and $A$-special, this chain is $A$-scattered. A one-to-one continuous reduction of $A$ to $H$ maps this $A$-scattered chain onto an $H$-scattered chain in $Q$.

$(i v) \Rightarrow(i)$. Fix some presentation $\left(b_{i}\right)_{i \in \mathbb{N}}$ of $P$. Let $\left(y_{\beta}\right)_{\beta \leq \alpha}$ be the $H$-scattered chain and $\theta_{\beta}$ 's, $\beta \leq \alpha$, the one-to-one continuous maps $\mathcal{P} \rightarrow P$ as in Definition 4.11. We keep the notations in the proof of the same implication in Theorem 4.8. Let $f: P \rightarrow Q$ be the continuous reduction of $X$ to $H$ constructed in this proof: $f(z)=y_{\tau(z)}$ where $\tau(z)$ is the least $\beta<\alpha$ such that $z \in B_{\beta}$ if $z \in \bigcup_{\beta<\alpha} V_{\beta}$ and $\tau(z)=\alpha$ otherwise. We introduce another map $g: P \rightarrow Q$ such that $g(z)=\theta_{\tau(z)}\left(\left\{i \mid b_{i} \ll z\right\}\right)$. Let us see that $g: P \rightarrow Q$ reduces $A$ to $H$. If $z \in A$ then $f(z)=y_{\tau(z)} \in H$. Suppose $\tau(z)<\alpha$. We know that $\theta_{\tau(z)}$ maps $\mathcal{P} \mathbb{N}$ into $\left\{y \mid y_{\tau(z)} \sqsupseteq y \sqsupset y_{\tau(z)+1}\right\}$. Since $y_{\tau(z)} \in H, \theta_{\tau(z)}$ maps $\mathcal{P} \mathbb{N}$ into $H$. In particular, $g(z)=\theta_{\tau(z)}\left(\left\{i \mid b_{i} \ll z\right\}\right) \in H$. The case $\tau(z)=\alpha$ is similar. If $z \notin A$ the proof can be treated similarly.

We now check that $g: P \rightarrow Q$ is one-to-one. Suppose $z, t \in P$ and $z \neq t$. If $\tau(z)<\tau(t)$ then $x_{\tau(z)} \sqsupseteq g(z) \sqsupset x_{\tau(z)+1} \sqsupseteq x_{\tau(t)} \sqsupseteq g(t)$ hence $g(z) \neq g(t)$. If $\tau(z)=\tau(t)$ then, for the same $\beta=\tau(z)$, we have $g(z)=\theta_{\beta}\left(\left\{i \mid b_{i} \ll z\right\}\right)$ and $g(t)=\theta_{\beta}\left(\left\{i \mid b_{i} \ll t\right\}\right)$. Since $z \neq t$ we have $\left\{i \mid b_{i} \ll z\right\} \neq\left\{i \mid b_{i} \ll t\right\}$. Since $\theta_{\beta}$ is one-to-one we have $g(z) \neq g(t)$. Let us now see that $g: P \rightarrow Q$ is continuous. It is clear that $g$ is increasing. Suppose $z=\sqcup_{n \in \mathbb{N}} z_{n}$ where $\left(z_{n}\right)_{n \in \mathbb{N}}$ is an increasing sequence of elements of $P$. Since $z \in V_{\tau(z)}$ and $V_{\tau(z)}$ is open, for $n$ large enough, $z_{n}$ is also in $V_{\tau(z)}$ hence $\tau\left(z_{n}\right) \leq \tau(z)$. Now, $\tau: P \rightarrow\{\beta \mid \beta \leq \alpha\}$ is decreasing so that $\tau\left(z_{n}\right) \geq \tau(z)$. Thus, we get equality $\tau\left(z_{n}\right)=\tau(z)$. Consequently, $g(z)$ and the $g\left(z_{n}\right)$ 's, for $n$ large enough, are of the form $\theta_{\beta}(z)$ and $\theta_{\beta}\left(z_{n}\right)$ for the same $\beta=\tau(z)$. Since $\theta_{\beta}$ is continuous we get $g(z)=\theta_{\beta}(z)=\sqcup_{n \in \mathbb{N}} \theta_{\beta}\left(z_{n}\right)=\sqcup_{n \in \mathbb{N}} g\left(z_{n}\right)$. This proves continuity of $g$.

Using Corollary 4.13, the implications $\left(i_{e}\right) \Rightarrow\left(i i_{e}\right) \Rightarrow\left(i i i_{e}\right) \Rightarrow\left(i v_{e}\right) \Rightarrow\left(i_{e}\right)$ are proved as in Theorem 4.8 .

The next Corollary was known for the domain $\mathcal{P N}$, (Selivanov 2005b), cf. Corollary 3.15.

Corollary 4.15. Let $Q$ be an $\omega$-continuous domain and $\alpha<\omega_{1}$ be a countable ordinal.

1. If $Q$ admits some scattered chain of length $\alpha+1$ then there exists a one-to-one Wadge complete set for $\mathbf{D}_{\alpha}(Q)$. In particular, this applies if $Q=\mathcal{P} \mathbb{N}$.

2. Suppose $Q$ is $\omega$-continuous and admits an effective presentation. Fix such an effective presentation. If $Q$ admits some computable scattered chain of length $\alpha+1$ then there exists a one-to-one Wadge complete set for $\mathbf{D}_{\alpha}(Q)$ which is also effectively Wadge complete for $\mathrm{D}_{\alpha}(Q)$. In particular, this applies if $Q=\mathcal{P} \mathbb{N}$.

Proof. Using Corollary 4.13 the proof is similar to that of Corollary 4.10. 
Wadge completeness does not coincide with one-to-one Wadge completeness in $\mathcal{P} \mathbb{N}$.

Corollary 4.16. There are Wadge complete families for $\mathbf{D}_{\alpha}(\mathcal{P N})$ which are not Wadge one-to-one complete.

Proof. Consider a partition $\left(A_{\beta}\right)_{\beta<\alpha}$ of $\mathbb{N}$ in infinite sets and let $A_{\alpha}=\varnothing$. Let $X_{\beta}=$ $\cup_{\beta \leq \delta \leq \alpha} A_{\delta}$ and $U_{\beta}$ be the sets that meet $\cup_{\gamma \leq \beta} A_{\gamma}$. Let $\mathcal{H}=D_{\alpha}\left(\left(U_{\beta}\right)_{\beta<\alpha}\right)$. Since $\left(X_{\beta}\right)_{\beta<\alpha}$ is a decreasing $\mathcal{H}$-alternating chain, Theorem 4.8 ensures that $\mathcal{H}$ is Wadge complete for $\mathbf{D}_{\alpha}(\mathcal{P N})$. Observe that $\cup_{\beta<\alpha} U_{\beta}=\mathcal{P} \mathbb{N} \backslash\{\varnothing\}$. Thus, every decreasing $\mathcal{H}$-alternating chain of length $\alpha+1$ has the empty set as last element hence it cannot be scattered. This shows that $\mathcal{H}$ cannot be Wadge one-to-one complete.

\subsection{Finite-to-one Wadge completeness and alternating decreasing chains}

In the case of the $\omega$-continuous domain $(\mathcal{P} \mathbb{N}, \subseteq)$ finite-to-one and one-to-one Wadge hardness coincide.

Theorem 4.17. Let $\mathcal{H} \subseteq \mathcal{P} \mathbb{N}$.

1. The following conditions are equivalent.

(i) $\mathcal{H}$ is finite-to-one Wadge hard for $\mathbf{D}_{\alpha}(\mathcal{P} \mathbb{N})$.

(ii) $\mathcal{H}$ is one-to-one Wadge hard for $\mathbf{D}_{\alpha}(P)$ for every $\omega$-continuous domain $P$.

2. Fix some presentation of $Q$. If $\alpha<\omega_{1}^{C K}$ the following conditions are equivalent.

$\left(i_{e}\right) \mathcal{H}$ is effectively finite-to-one Wadge hard for $\mathrm{D}_{\alpha}(\mathcal{P} \mathbb{N})$.

$\left(i i_{e}\right) \mathcal{H}$ is one-to-one Wadge hard for $\mathbf{D}_{\alpha}(P)$ and effectively one-to-one Wadge hard for $\mathrm{D}_{\alpha}(P)$ for every effective $\omega$-continuous domain (cf. Definition 2.9).

Proof. $(i) \Rightarrow($ ii $)$. Consider the family $\mathcal{A} \in \mathbf{D}_{\alpha}(\mathcal{P N})$ and the $\mathcal{A}$-special chain $\left(X_{\beta}\right)_{\beta \leq \alpha}$ in $\mathcal{P} \mathbb{N}$ given by Proposition 4.4. Consider bijections $\mu_{\alpha}: \mathbb{N} \rightarrow X_{\alpha}$ and, for $\beta<\alpha$, $\mu_{\beta}: \mathbb{N} \rightarrow X_{\beta} \backslash X_{\beta+1}$. Let $X_{\alpha, n}=\mu_{\alpha}(\{0, \ldots, n\})$ and, for $\beta<\alpha$, let $X_{\beta, n}=X_{\beta+1} \cup \mu_{\beta}(\{0, \ldots, n\})$. Let $f: \mathcal{P N} \rightarrow \mathcal{P} \mathbb{N}$ be a finite-to-one continuous reduction of $\mathcal{A}$ to $\mathcal{H}$ and let $Z_{\beta}=f\left(X_{\beta}\right)$, for $\beta \leq \alpha$. Consider some $\beta<\alpha$. The sequence $\left(X_{\beta, n}\right)_{n \in \mathbb{N}}$ is an infinite chain in $\mathcal{P} \mathbb{N}$ of sets containing $X_{\beta+1}$ and contained in $X_{\beta}$. Since $f$ is increasing we have $Z_{\beta}=f\left(X_{\beta}\right) \supseteq$ $f\left(X_{\beta, n}\right) \supseteq f\left(X_{\beta+1}\right)=Z_{\beta+1}$. Since $f$ is finite-to-one, $\left(f\left(X_{\beta, n}\right)\right)_{n \in \mathbb{N}}$ contains infinitely many distinct sets. In particular, $Z_{\beta} \backslash Z_{\beta+1}$ must be an infinite set. A similar argument shows that $Z_{\alpha}$ is also an infinite set. Let $\theta_{\alpha}: \mathcal{P N} \rightarrow \mathcal{P N}$ be such that $\theta_{\alpha}(X)=\mu_{\alpha}(X)$ for all $X \in \mathcal{P} \mathbb{N}$. For $\beta<\alpha$, let $\theta_{\beta}: \mathcal{P} \mathbb{N} \rightarrow \mathcal{P} \mathbb{N}$ be such that $\theta_{\alpha}(X)=f\left(X_{\beta+1} \cup \mu_{\beta}(X)\right)$. These maps $\theta_{\beta}, \beta \leq \alpha$, are continuous one-to-one maps $\mathcal{P} \mathbb{N} \rightarrow \mathcal{P} \mathbb{N}$. Clearly, $\theta_{\alpha}(\varnothing)=\varnothing$ and $\theta_{\alpha}(\mathbb{N})=X_{\alpha}$. And for $\beta<\alpha, \theta_{\beta}(\varnothing)=Z_{\beta+1}$ and $\theta_{\beta}(\mathbb{N})=Z_{\beta}$. Recall that $\left(X_{\beta}\right)_{\beta \leq \alpha}$ is $\mathcal{A}$-special. Thus, for all $X \in \mathcal{P} \mathbb{N}$, the set $\mu_{\alpha}(X)$, being included in $X_{\alpha}$, is not in $\mathcal{A}$. For $\beta<\alpha$, the set $X_{\beta+1} \cup \mu_{\beta}(X)$, being included in $X_{\beta}$ and containing strictly $X_{\beta+1}$, is in $\mathcal{A}$ if and only if $\beta \psi \alpha$. Since $f$ is a reduction of $\mathcal{A}$ to $\mathcal{H}$, the range of $\theta_{\alpha}$ is included in $\mathcal{P} \mathbb{N} \backslash \mathcal{H}$ and, for $\beta<\alpha$, the range of $\theta_{\beta}$ is included in $\mathcal{H}$ if $\beta \psi \alpha$, and it is included in $\mathcal{P} \backslash \mathcal{H}$ if $\beta \sim \alpha$. This proves that the chain $\left(Z_{\beta}\right)_{\beta \leq \alpha}$ is $\mathcal{H}$-scattered. Applying Theorem 4.14, we get the wanted condition (ii).

$\left(i_{e}\right) \Rightarrow\left(i i_{e}\right)$. Routine variation of the above argument.

$(i i) \Rightarrow(i)$ and $\left(i i_{e}\right) \Rightarrow\left(i_{e}\right)$ are obvious. 


\subsection{Increasing chains of Wadge degrees}

Combining a construction given by Selivanov (2005b) with decreasing chains of sets, we get increasing chains of proper $\mathbf{D}_{\alpha}(\mathcal{P N})$ Wadge degrees. We write $\mathbb{N}^{<\omega}$ for the set of finite sequences of natural numbers, and $\mathbb{N} \leq \omega$ for $\mathbb{N}^{<\omega} \cup \mathbb{N}^{\omega}$. nil denotes the empty sequence.

\section{Definition 4.18 (Selivanov 2005b).}

Suppose $\alpha$ is infinite. Let $T \subset \mathbb{N}^{\leq \omega}$ be a well-founded tree (i.e. $T$ is closed under prefix and has no infinite branch) with rank $\alpha$. Let $\xi: \mathbb{N}^{<\omega} \rightarrow 2 \mathbb{N}$ be an injective map such that, $\xi($ nil $)=0$. Let $e: \mathbb{N}^{<\omega} \rightarrow \mathcal{P}_{<\omega}(2 \mathbb{N})$ be the map such that, for every $\sigma \in T$, $e(\sigma)=\{\xi(\tau) \mid \tau$ prefix of $\sigma\}$. Let $B(T)=\{X \in \mathcal{P} \mathbb{N} \mid \forall \sigma \in T \quad X \nsubseteq e(\sigma)\}$. And, for each ordinal $\alpha$, let $Y_{\alpha}=\{e(\sigma) \mid \sigma \in T$ has odd length $\}$ and $Z_{\alpha}=Y_{\alpha} \cup B(T)$.

\section{Theorem 4.19 (Selivanov 2005b, Lemma 5.8 and Propositions 5.9 and 6.4).}

$B(T)$ is open and $Y_{\alpha}, Z_{\alpha}$ are two proper $\mathbf{D}_{\alpha}(\mathcal{P} \mathbb{N})$ families that are Wadge incomparable.

Before entering the construction of the chains of Wadge degrees, a simple lemma.

Lemma 4.20. Let $E$ be a topological space.

1. If $A \in \mathbf{D}_{\alpha}(E)$ and $L \subseteq E$ is open then $A \cap L \in \mathbf{D}_{\alpha}(E)$.

2. If $\alpha$ is infinite, $A \in \mathbf{D}_{\alpha}(E)$ and $F \subseteq E$ is closed then $A \cap F \in \mathbf{D}_{\alpha}(E)$.

3. Suppose $\beta>0, \beta+\alpha=\alpha$ (i.e. $\alpha \geq \beta \omega$ ). If $C \in \mathbf{D}_{\beta}(E), D \in \mathbf{D}_{\alpha}(E), L$ is open and $C \subseteq L$ and $L$ and $D$ are disjoint then $C \cup D \in \mathbf{D}_{\alpha}(E)$.

Proof. 1. If $A=D_{\alpha}\left(\left(U_{\beta}\right)_{\beta<\alpha}\right)$ then $A \cap L=D_{\alpha}\left(\left(U_{\beta} \cap L\right)_{\beta<\alpha}\right)$.

2. Let $L=E \backslash F$. Let $A=D_{\alpha}\left(\left(U_{\beta}\right)_{\beta<\alpha}\right)$ where the $U_{\beta}$ 's are open. In case $\alpha$ is odd, let $X_{0}=\varnothing, X_{1}=L$ and, for $\beta<\alpha$, let $X_{2+\beta}=L \cup U_{\beta}$. In case $\alpha$ is even, let $X_{0}=L$ and $X_{1+\beta}=L \cup U_{\beta}$. Now, $2+\alpha=1+\alpha=\alpha$ (since $\alpha$ is infinite) and $A \cap F=D_{\alpha}\left(\left(X_{\beta}\right)_{\beta<\alpha}\right)$.

3. Suppose $C=D_{\beta}\left(\left(U_{\gamma}\right)_{\gamma<\beta}\right)$ and $D=D_{\alpha}\left(\left(V_{\delta}\right)_{\delta<\alpha}\right)$. Case $\beta \sim \alpha$. Let $W_{\gamma}=U_{\gamma} \cap L$ for $\gamma<\beta, W_{\beta+\delta}=V_{\delta} \cup L$ for $\delta<\alpha$. Then,

$$
\begin{aligned}
D_{\alpha}\left(\left(W_{\gamma}\right)_{\gamma<\alpha}\right) & =D_{\beta}\left(\left(U_{\gamma} \cap L\right)_{\gamma<\beta}\right) \cup\left(D_{\alpha}\left(\left(V_{\delta} \cup L\right)_{\beta \leq \delta<\alpha}\right) \backslash \cup_{\gamma<\beta} U_{\gamma} \cap L\right) \\
& =(C \cap L) \cup\left((D \backslash L) \backslash \cup_{\gamma<\beta} U_{\gamma} \cap L\right) \\
& =(C \cap L) \cup(D \backslash L) \\
& =C \cup D .
\end{aligned}
$$

Case $\beta \neq \alpha$. Let $W_{0}=\varnothing, W_{1+\gamma}=U_{\gamma} \cap L$ for $\gamma<\beta, W_{\beta+\delta}=V_{\delta} \cup L$ for $\delta<\alpha$, and argue similarly.

Definition 4.21. Let $\left(a_{\delta}\right)_{\delta<\alpha}$ be a one-to-one enumeration of $2 \mathbb{N}+3$. For $\beta<\alpha$ let $U_{\beta}$ be the open family of sets containing 1 and meeting $\left\{a_{\gamma} \mid \gamma \leq \beta\right\}$. Let $\mathcal{A}=D_{\alpha}\left(\left(U_{\delta}\right)_{\delta<\alpha}\right) \in \mathbf{D}_{\alpha}(\mathcal{P} \mathbb{N})$. For $\beta<\alpha$, set $\mathcal{A}_{\beta}=D_{\beta}\left(\left(U_{\delta}\right)_{\delta<\beta}\right) \cap \mathcal{P}(2 \mathbb{N}+1)$. Let $Y_{\alpha}$ as in Definition 4.18 and let $Y_{\alpha, \beta}=\mathcal{A}_{\beta} \cup Y_{\alpha}$ and $Z_{\alpha, \beta}=\left(B(T) \cap \mathcal{B}_{\{0\}}\right) \cup Y_{\alpha, \beta}$.

The following result is straightforward.

\section{Proposition 4.22.}

1. $Y_{\alpha, \beta}$ is included in $\mathcal{P}(2 \mathbb{N}) \cup \mathcal{P}(2 \mathbb{N}+1)$.

2. A set $X$ is in $\mathcal{A}$ if and only if $1 \in X$ and $X$ meets $2 \mathbb{N}+3$ and the least $\delta$ such that $a_{\delta} \in X$ is such that $\delta \psi \alpha$. 
3. A set $X$ is in $\mathcal{A}_{\beta}$ if and only if $1 \in X, X \subseteq 2 \mathbb{N}+1$ and $X$ meets $\left\{a_{\delta} \mid \delta<\beta\right\}$ and the least $\delta$ such that $a_{\delta} \in X$ is such that $\delta \neq \beta$.

4. For $\beta \leq \alpha$, set $X_{\beta}=\left\{a_{\delta} \mid \delta \geq \beta\right\} \cup\{1\}$. The sequence $\left(X_{\delta}\right)_{\delta \leq \alpha}$ is a decreasing $\mathcal{A}$-alternating chain. The sequence $\left(X_{\delta}\right)_{\delta \leq \beta}$ is alternating relative to $Y_{\alpha, \beta}$ and to $Z_{\alpha, \beta}$.

5. If $X \in B(T)$ (cf. Definition 4.18) then $(X \cap 2 \mathbb{N}) \cup I \in B(T)$ for every $I \subseteq 2 \mathbb{N}+1$.

\section{Theorem 4.23.}

1. If $\omega \leq \beta+\alpha=\alpha$ then $Y_{\alpha, \beta}$ and $Z_{\alpha, \beta}$ are proper families in $\mathbf{D}_{\alpha}(\mathcal{P} \mathbb{N})$.

2. If $\omega \leq \beta<\gamma<\alpha$ then $Y_{\alpha, \beta}<_{W} Y_{\alpha, \gamma}$ and $Z_{\alpha, \beta}<_{W} Z_{\alpha, \gamma}$.

3. If $\omega \leq \beta, \delta$ and $\beta, \delta<\alpha$ then $Y_{\alpha, \beta}$ and $Z_{\alpha, \delta}$ are Wadge incomparable.

4. For $\alpha<\omega_{1}^{C K}, Y_{\alpha, \beta}$ and $Z_{\alpha, \delta}$ are proper families in $\mathrm{D}_{\alpha}(\mathcal{P} \mathbb{N})$, and the effective counterparts of items 2 and 3 hold.

Proof. 1. First, we show that $Y_{\alpha, \beta} \in \mathbf{D}_{\alpha}(\mathcal{P N})$. Since $\mathcal{P}(2 \mathbb{N}+1)$ is closed in $\mathcal{P}$, item 2 of Lemma 4.20 ensures that $\mathcal{A}_{\beta} \in \mathbf{D}_{\beta}(\mathcal{P} \mathbb{N})$. Now, $\mathcal{A}_{\beta}$ is included in the open family $\mathcal{B}_{\{1\}}$ which is disjoint from $Y_{\alpha}$ since all sets in $Y_{\alpha} \subset \mathcal{P}(2 \mathbb{N})$. Item 3 of Lemma 4.20 ensures that $Y_{\alpha, \beta}=\mathcal{A}_{\beta} \cup Y_{\alpha} \in \mathbf{D}_{\alpha}(\mathcal{P} \mathbb{N})$. We now prove that $Z_{\alpha, \beta} \in \mathbf{D}_{\alpha}(\mathcal{P} \mathbb{N})$. The family $Y_{\alpha}$ is disjoint from $B(T)$ hence from $B(T) \cap \mathcal{B}_{\{0\}}$. Also, $B(T) \cap \mathcal{B}_{\{0\}}$ is disjoint from $\mathcal{A}_{\beta}$ since every set in $B(T) \cap \mathcal{B}_{\{0\}}$ contains 0 whereas all sets in $\mathcal{A}_{\beta}$ are included in $2 \mathbb{N}+1$. Thus, $B(T) \cap \mathcal{B}_{\{0\}}$ is disjoint from $Y_{\alpha, \beta}$. Since $B(T) \cap \mathcal{B}_{\{0\}}$ is open, applying again item 3 of Lemma 4.20, we see that $Z_{\alpha, \beta}=\left(B(T) \cap \mathcal{B}_{\{0\}}\right) \cup Y_{\alpha, \beta}$ is in $\mathbf{D}_{\alpha}(\mathcal{P N})$. Finally, notice that the map $f: X \mapsto(X \cap 2 \mathbb{N}) \cup\{2 n+3 \mid 2 n+1 \in X\}$ is continuous and reduces $Y_{\alpha}$ to $Y_{\alpha, \beta}$. The map sending $X$ to $f(X)$ if $X \notin B(T)$ and to $f(X) \cup\{0\}$ if $X \in B(T)$ is also continuous and reduces $Z_{\alpha}$ to $Z_{\alpha, \beta}$. Since $Y_{\alpha}$ and $Z_{\alpha}$ are outside $c o-\mathbf{D}_{\alpha}(\mathcal{P} \mathbb{N})$ (cf. Theorem 4.19) so are $Y_{\alpha, \beta}$ and $Z_{\alpha, \beta}$.

2. The reducibilities $Y_{\alpha, \beta} \leq_{W} Y_{\alpha, \gamma}$ and $Z_{\alpha, \beta} \leq_{W} Z_{\alpha, \gamma}$ can be both witnessed by a same continuous map $f: \mathcal{P} \mathbb{N} \rightarrow \mathcal{P}$,

$$
\left\{\begin{aligned}
\text { if } \beta \psi \gamma \text { then } f(X)= & (X \cap 2 \mathbb{N}) \cup\left\{a_{\delta+1} \mid \delta<\beta \text { and } a_{\delta} \in X\right\} \cup(X \cap\{1\}) \\
\text { if } \beta \sim \gamma \text { then } f(X)= & (X \cap 2 \mathbb{N}) \cup\left(X \cap\left(\left\{a_{\delta} \mid \delta<\beta\right\} \cup\{1\}\right)\right) \cup M, \\
& \text { where } M=\left\{a_{\beta}\right\} \text { if } X \text { meets } 2 \mathbb{N}+1 \text { and } M=\varnothing \text { otherwise. }
\end{aligned}\right.
$$

We now show that the reducibilities are strict. Towards a contradiction, suppose that there is a reduction $f$ from $Y_{\alpha, \gamma}$ to $Y_{\alpha, \beta}$. Then the decreasing $Y_{\alpha, \gamma}$-alternating chain $\left(X_{\delta}\right)_{\delta \leq \gamma}$ would be mapped onto a $Y_{\alpha, \beta}$-alternating chain $\left(f\left(X_{\delta}\right)\right)_{\delta \leq \gamma}$ (recall that by item 4 of Proposition 4.22, $\left.X_{\beta}=\left\{a_{\delta} \mid \delta \geq \beta\right\} \cup\{1\}\right)$. Let $\varepsilon \in\{0,1\}$ be least such that $f\left(X_{\varepsilon}\right) \in Y_{\alpha, \beta}$. Since $Y_{\alpha, \beta} \subset \mathcal{P}(2 \mathbb{N}) \cup \mathcal{P}(2 \mathbb{N}+1)$, we have $f\left(X_{\varepsilon}\right) \in \mathcal{P}(2 \mathbb{N}) \cup \mathcal{P}(2 \mathbb{N}+1)$. The chain being strictly decreasing, we see that the family $\left\{f\left(X_{\delta}\right) \mid \varepsilon \leq \delta \leq \gamma\right\}$ is included either in $\mathcal{P}(2 \mathbb{N})$ or in $\mathcal{P}(2 \mathbb{N}+1)$. Now, $Y_{\alpha, \beta} \cap \mathcal{P}(2 \mathbb{N})=Y_{\alpha}$ contains no infinite decreasing chain. Thus, $\left\{f\left(X_{\delta}\right) \mid \varepsilon \leq \delta \leq \gamma\right\} \subseteq \mathcal{P}(2 \mathbb{N}+1)$. Since the chain $\left(f\left(X_{\delta}\right)\right)_{2 \leq \delta \leq \gamma}$ is also $Y_{\alpha, \beta}$-alternating and has length $\gamma+1$ (since $\gamma$ is infinite), this contradicts the fact that $Y_{\alpha, \beta} \cap \mathcal{P}(2 \mathbb{N}+1)=\mathcal{A}_{\beta}$ does not contain alternating chains longer than $\beta+1$. To see that $Z_{\alpha, \beta}<_{W} Z_{\alpha, \gamma}$, observe that $B(T)$ is an upset, hence it has no infinite decreasing alternating chain. Then argue as for $Y_{\alpha, \gamma} \nless_{W} Y_{\alpha, \beta}$.

3. Let us check that $Y_{\alpha, \beta} \Varangle_{W} Z_{\alpha, \delta}$. Towards a contradiction, suppose that $f$ reduces $Y_{\alpha, \beta}$ to $Z_{\alpha, \delta}$. Since $\mathbb{N} \notin Y_{\alpha, \beta}$ we have $f(\mathbb{N}) \notin Z_{\alpha, \beta}$. In particular, $f(\mathbb{N}) \notin B(T) \cap \mathcal{B}_{\{0\}}$. 
Hence, either $f(\mathbb{N}) \notin B(T)$ or $0 \notin f(\mathbb{N})$. Suppose $f(\mathbb{N}) \notin B(T)$. Then $f(\mathbb{N}) \subseteq e(\sigma)$ for some $\sigma \in T$. In particular, $f(\mathbb{N})$ is finite hence has finitely many subsets. This contradicts the fact that the infinite decreasing $Y_{\alpha, \beta}$-alternating chains are mapped by $\mathrm{f}$ to infinite decreasing $Z_{\alpha, \delta}$-alternating chains below $f(\mathbb{N})$. If, instead, $0 \notin f(N)$, then $0 \notin f(X)$ (for all $X$ ) hence $f$ reduces $Y_{\alpha, \beta}$ to $Y_{\alpha, \delta}=\mathcal{A}_{\delta} \cup Y_{\alpha}$ hence to $\mathcal{A}_{\delta}$ (since all sets in $Y_{\alpha}$ contain 0 whereas no $f(X)$ does). This is already a contradiction since $\delta<\alpha, Y_{\alpha, \beta}$ is a proper $\mathrm{D}_{\alpha}(\mathcal{P N})$ set by item 1 , and $A_{\delta}$ is a $\mathrm{D}_{\delta}(\mathcal{P N})$ set by the proof of item 1 . Finally, we check that $Z_{\alpha, \delta} \Varangle_{W} Y_{\alpha, \beta}$. Since $\mathbb{N} \in Z_{\alpha, \delta}$ (it is in $B(T)$ ) we have $f(\mathbb{N}) \in Y_{\alpha, \beta}$. If $f(\mathbb{N}) \in Y_{\alpha}$ then $f(\mathbb{N})$ is finite. This contradicts the existence of infinite alternating decreasing chains. Thus, $f(\mathbb{N}) \in \mathcal{A}_{\beta}$ hence $f(\mathbb{N})$ is included in $2 \mathbb{N}+1$. Since $f$ is increasing, $f$ takes values in $\mathcal{P}(2 \mathbb{N}+1)$. Thus, $f$ reduces $Z_{\alpha, \delta}$ to $A_{\beta}$. But $\beta<\alpha, Z_{\alpha, \delta}$ is a proper $\mathrm{D}_{\alpha}(\mathcal{P} \mathbb{N})$ set by item 1 , and $A_{\beta}$ is a $\mathrm{D}_{\beta}(\mathcal{P} \mathbb{N})$ set by the proof of item 1 , the same contradiction as before.

4. Since $\alpha<\omega_{1}^{C K}$, the tree $T$ in Definition 4.18 can be taken computable. Then $Y_{\alpha}$ is a computable subfamily of $\mathcal{P}_{<\omega} \mathbb{N}$ and $B(T)$ is an effective open family. We can take a computable enumeration $\left(a_{\delta}\right)_{\delta<\alpha}$ as in Definition 4.21. Thus, the $Y_{\alpha, \beta}$ 's and $Z_{\alpha, \gamma}$ 's are in $\mathrm{D}_{\alpha}(\mathcal{P N})$. To see that for $\beta<\gamma, Y_{\alpha, \beta}$ is effectively reducible to $Y_{\alpha, \gamma}$, and $Z_{\alpha, \beta}$ is effectively reducible to $Z_{\alpha, \gamma}$, observe that the map $f$ defined in the proof of item 2 is effective.

\section{Wadge hardness in $\mathcal{P} \mathbb{N}$ versus Wadge hardness in other spaces}

Due to the universality property of $\mathcal{P} \mathbb{N}$ (cf. Proposition 3.14), Wadge hardness relative to a Borel class of $\mathcal{P} \mathbb{N}$ is stronger than Wadge hardness relative to the same Borel class of many other spaces.

\subsection{Simple Wadge complete sets in $\mathcal{P} \mathbb{N}$ for $\boldsymbol{\Sigma}_{2}^{0}$}

Definition 5.1. Let $\mathcal{S}_{2}=\{X \in \mathcal{P} \mathbb{N} \mid \exists n(2 n \in X \wedge 2 n+1 \notin X)\}$.

Proposition 5.2. The family $\mathcal{S}_{2}$ is Wadge complete for $\Sigma_{2}^{0}(\mathcal{P N})$ and effectively Wadge complete for $\Sigma_{2}^{0}(\mathcal{P N})$.

Proof. From its definition it is clear that $\mathcal{S}_{2}$ is $\Sigma_{2}^{0}(\mathcal{P N})$. Consider a $\Sigma_{2}^{0}(\mathcal{P} \mathbb{N})$ family $\mathcal{X}=\bigcup_{n \in \mathbb{N}}\left\{Z \mid \exists A \in \mathcal{A}_{n} Z \supseteq A\right\} \backslash\left\{Z \mid \exists B \in \mathcal{B}_{n} Z \supseteq B\right\}$, where the $\mathcal{A}_{n}$ 's and $\mathcal{B}_{n}$ 's are families of finite sets. Define $f: \mathcal{P N} \rightarrow \mathcal{P N}$ as

$$
f(X)=\left\{2 n \mid \exists A \in \mathcal{A}_{n} A \subseteq X\right\} \cup\left\{2 n+1 \mid \exists B \in \mathcal{B}_{n} B \subseteq X\right\} .
$$

Then, $X \in \mathcal{X} \Leftrightarrow \exists n(2 n \in f(X) \wedge 2 n+1 \notin f(X)) \Leftrightarrow f(X) \in \mathcal{S}_{2}$. Observe that $f$ is continuous hence it is a reduction of $\mathcal{X}$ to $\mathcal{S}_{2}$. In case $\mathcal{X}$ is $\Sigma_{2}^{0}(\mathcal{P N})$ then the $\mathcal{A}_{n}, \mathcal{B}_{n}$ are uniformly computably enumerable so that $f$ is effective.

\subsection{Alternating increasing chains for $\boldsymbol{\Sigma}_{2}^{0}(\mathcal{P} \mathbb{N})$ families}

As stated in Remark 4.3, in $\omega$-continuous domains there are no increasing $A$-alternating chains of length $\omega$ when $A$ is $\Delta_{2}^{0}$. This is not true anymore for $A$ in $\Sigma_{2}^{0}$. 
Proposition 5.3. For every ordinal $\alpha<\omega_{1}$, there exists in $\mathcal{P} \mathbb{N}$ an $\mathcal{S}_{2}$-alternating increasing chain with length $\alpha$. If $\alpha<\omega_{1}^{C K}$ then the sequence can be taken computable.

Proof. Let $\left(a_{\beta}\right)_{\beta<\alpha}$ be an injective enumeration of $\mathbb{N}$. Let

$$
X_{2 \beta}=\left\{2 a_{\gamma}, 2 a_{\gamma}+1 \mid \gamma<\beta\right\} \cup\left\{2 a_{\beta}\right\} \text { and } X_{2 \beta+1}=X_{2 \beta} \cup\left\{2 a_{\beta}+1\right\} .
$$

Clearly, $\left(X_{\beta}\right)_{\beta<\alpha}$ is increasing, and for each $\beta<\alpha, X_{2 \beta} \in \mathcal{S}_{2}$ but $X_{2 \beta+1}$ is not.

Remark 5.4. As a corollary, we see that the space $\mathbf{2}^{\leq \omega}$, where all increasing chains have length at most $\omega+1$, contains no Wadge hard set for $\Sigma_{2}^{0}(\mathcal{P} \mathbb{N})$.

The above proposition can be improved. We first define a notion of alternating chain.

Definition 5.5. Let $(E, \leq)$ be a linear ordering and $A \subseteq E$. Let $(P, \subseteq)$ be a partially ordered set and $H \subseteq P$.

1. $A$ is an alternation on $E$ if, for all $a, b \in E$, the following conditions are satisfied.

- If $b$ is successor to $a$ then $a \in A$ if and only if $b \notin A$.

- If $a$ has no predecessor nor successor then $a \notin A$.

2. An $(H, A)$-alternating $E$-chain in $P$ is an $E$-sequence $\left(x_{a}\right)_{a \in E}$ of elements of $P$ that satisfies the following conditions: for all $a, b \in E$,

$-a \leq b$ (in the linear order $E$ ) if and only if $x_{a} \sqsubseteq x_{b}$ (in the partial order $P$ ),

- $x_{a} \in H$ if and only if $a \in A$.

We now define a very rich linear order with respect to alternation.

Definition 5.6. Let $(\mathcal{R}, \leq)$ be the totally ordered set obtained from $\mathbb{R}$ by replacing each rational number by a chain of type $\mathbb{Z}$. Formally, $\mathcal{R}=(\mathbb{R} \backslash \mathbb{Q}) \cup(\mathbb{Q} \times \mathbb{Z})$ with the following order: for $x \in \mathbb{R} \backslash \mathbb{Q}, q, q^{\prime} \in \mathbb{Q}, z, z^{\prime} \in \mathbb{Z}$,

$$
x<(q, z) \Leftrightarrow x<q, \quad(q, z)<x \Leftrightarrow q<x, \quad(q, z)<\left(q^{\prime}, z^{\prime}\right) \Leftrightarrow q<q^{\prime} \text { or }\left(q=q^{\prime} \text { and } z<z^{\prime}\right) .
$$

Remark 5.7. The above linear order is very rich in the following sense. In a linear order $(E, \leq)$ let $\sim_{E}$ be the equivalence relation such that $x \sim_{E} y$ if and only if there are finitely many elements between $x$ and $y$ (i.e. we identify an element with its successor if there is one). If $(E, \leq)$ has countably many successor pairs and its quotient is embedded in $\mathbb{R}$ then $(E, \leq)$ is embedded in $\mathcal{R}$.

Proposition 5.8. Le $P$ be an $\omega$-continuous domain and $H \subseteq P$.

1. If $H$ is Wadge hard for $\Sigma_{2}^{0}(\mathcal{P N})$ then $P$ contains an $(H, \mathcal{A})$-alternating $\mathcal{R}$-chain for every alternation $\mathcal{A}$ on $\mathcal{R}$ (cf. Definition 5.6). In particular, the set $P \backslash H$ has cardinality $2^{\aleph_{0}}$.

2. Suppose $P$ is an $\omega$-continuous domain and fix some presentation $\left(p_{i}\right)_{i \in \mathbb{N}}$ of $P$. If $H \subseteq P$ is effectively Wadge hard for $\Sigma_{2}^{0}(\mathcal{P} \mathbb{N})$ then, for every alternation $\mathcal{A}$ on $\mathcal{R}$ which is computable as a subset of $\mathbb{Q} \times \mathbb{Z}, P$ contains an $(H, \mathcal{A})$-alternating $\mathcal{R}$-chain $\left(u_{\xi}\right)_{\xi \in \mathcal{R}}$ such that the sets

$$
\left\{(q, n, i) \mid q \in \mathbb{Q}, n \in \mathbb{Z}, p_{i} \ll u_{(q, n)}\right\} \text { and }\left\{(q, i) \mid q \in \mathbb{Q}, \forall x \in \mathbb{R} \backslash \mathbb{Q}\left(q<x \Rightarrow p_{i} \ll u_{x}\right)\right\}
$$

are both computably enumerable. In particular, the set $P \backslash H$ has cardinality $2^{\aleph_{0}}$. 
Note 5.9. It is known that any Borel subset of a Polish space is either countable or has cardinality $2^{\aleph_{0}}$. The same is true in any quasi-Polish space, hence in any $\omega$-continuous domain, since its Borel sets are those of a Polish topology on the same space (de Brecht 2011). Since the Cantor space $\mathbf{2}^{\omega}$ is $T_{1}$, any singleton set is closed. Hence, any set with countable complement is in $\boldsymbol{\Pi}_{2}^{0}\left(\mathbf{2}^{\omega}\right)$ and it cannot be Wadge hard for $\boldsymbol{\Sigma}_{2}^{0}\left(\mathbf{2}^{\omega}\right)$. This easy argument, showing that the complement of any set $A \subseteq \mathbf{2}^{\omega}$ which is Wadge hard for $\boldsymbol{\Sigma}_{2}^{0}\left(\mathbf{2}^{\omega}\right)$ has cardinality $2^{\aleph_{0}}$, breaks down in the Scott domain $\mathcal{P} \mathbb{N}$. If $X \in \mathcal{P} \mathbb{N}, X \neq \varnothing$ then the singleton family $\{X\}$ is in $\Pi_{2}^{0}(\mathcal{P N})$ but it is not closed in $\mathcal{P} \mathbb{N}$. Thus, a family with a countable complement need not to be in $\Pi_{2}^{0}(\mathcal{P N})$.

Proof of Proposition 5.8. 1. For every alternation $\mathcal{A}$ on $\mathcal{R}$, we construct in $\mathcal{P} \mathbb{N}$ an $\left(\mathcal{S}_{2}, \mathcal{A}\right)$-alternating $\mathcal{R}$-chain $\left(X_{\xi}\right)_{\xi \in \mathcal{R}}$. For every $q \in \mathbb{Q}$, let $\varepsilon_{q}=0$ if $(q, 0) \in \mathcal{A}$ and $\varepsilon_{q}=1$ if $(q, 0) \notin \mathcal{A}$. Let $\left(E_{q}\right)_{q \in \mathbb{Q}}$ be a partition of $2 \mathbb{N}$ into infinite sets. For every $q \in \mathbb{Q}$, let $\varphi_{q}: \mathbb{Z} \rightarrow E_{q}$ be some bijective map. The $\mathcal{R}$-chain is defined as follows.

For $q \in \mathbb{Q}, n \in \mathbb{Z}, x \in \mathbb{R} \backslash \mathbb{Q}$,

$$
\begin{aligned}
X_{\left(q, 2 n+\varepsilon_{q}\right)} & =\left(\cup_{r \in \mathbb{Q}, r<q} E_{r}\right) \cup\left\{\varphi_{q}(p), \varphi_{q}(p)+1 \mid p \in \mathbb{Z}, p<n\right\} \\
X_{\left(q, 2 n+\varepsilon_{q}+1\right)} & =X_{(q, 2 n)} \cup\left\{\varphi_{q}(n)\right\} \\
X_{x} & =\bigcup_{q \in \mathbb{Q}, q<x} E_{q} .
\end{aligned}
$$

It is easy to check that this chain is $\left(\mathcal{S}_{2}, \mathcal{A}\right)$-alternating. Suppose now that $H \subseteq P$ is Wadge hard for $\Sigma_{2}^{0}(\mathcal{P N})$. Let $f: \mathcal{P} \mathbb{N} \rightarrow P$ be continuous such that $f^{-1}(H)=\mathcal{S}_{2}$. Then $f$ maps this $\left(\mathcal{S}_{2}, \mathcal{A}\right)$-alternating $\mathcal{R}$-chain onto an $(H, \mathcal{A})$-alternating $\mathcal{R}$-chain in $P$. If $x, y \in \mathbb{R} \backslash \mathbb{Q}$ and $x<y$ then $X_{x} \subset X_{q, 0} \subset X_{q, 1} \subset X_{y}$. Applying the reduction $f$, we get $f\left(X_{x}\right) \sqsubseteq f\left(X_{q, 0}\right) \sqsubseteq$ $f\left(X_{q, 1}\right) \subseteq f\left(X_{y}\right)$. Since $X_{q, 0} \in \mathcal{S}_{2} \Leftrightarrow X_{q, 1} \notin \mathcal{S}_{2}$ we have $f\left(X_{q, 0}\right) \in H \Leftrightarrow f\left(X_{q, 1}\right) \notin H$ hence $f\left(X_{q, 0}\right) \sqsubset f\left(X_{q, 1}\right)$ and $f\left(X_{x}\right) \sqsubset f\left(X_{y}\right)$. Thus, $P \backslash H$ has cardinality $2^{\omega}$ since it contains the pairwise distinct elements $f\left(X_{x}\right)$ 's, $x \in \mathbb{R} \backslash \mathbb{Q}$.

2. Observe that the map $q \mapsto \varepsilon_{q}$ is computable and take a computable partition $\left(E_{q}\right)_{q \in \mathbb{Q}}$ and a computable sequence of bijections $\varphi_{q}$.

\subsection{Classical families hard for $\boldsymbol{\Sigma}_{2}^{0}\left(\mathbf{2}^{\omega}\right)$ but not hard for $\boldsymbol{\Sigma}_{2}^{0}(\mathcal{P} \mathbb{N})$}

A priori, modulo the obvious bijection between the Cantor space $\mathbf{2}^{\omega}$ and $\mathcal{P N}$, families of $\mathbf{2}^{\omega}$ that are Wadge hard for a class in $\mathbf{2}^{\omega}$ may seem good candidates to be also hard families for the same class in $\mathcal{P} \mathbb{N}$. This is not the case. We state here some counterexamples. For results about the Wadge hardness of classical $\Sigma_{3}^{0}(\mathcal{P N})$ families for various classes and spaces, we refer the reader to Becher and Grigorieff (2009).

Theorem 5.10. Let $\theta: \mathcal{P} \mathbb{N} \rightarrow \mathbf{2}^{\omega}$ be the bijection which maps a set to its characteristic function. Then,

1. $\theta\left(\mathcal{P}_{<\omega} \mathbb{N}\right)$ is Wadge $\boldsymbol{\Sigma}_{2}^{0}\left(\mathbf{2}^{\omega}\right)$-complete and effectively Wadge $\Sigma_{2}^{0}\left(\mathbf{2}^{\omega}\right)$-complete. In particular, $\theta\left(\mathcal{P}_{<\omega} \mathbb{N}\right)$ is in $\Sigma_{2}^{0}\left(\mathbf{2}^{\omega}\right) \backslash \boldsymbol{\Pi}_{2}^{0}\left(\mathbf{2}^{\omega}\right)$.

2. $\mathcal{P}_{<\omega} \mathbb{N} \in \Sigma_{2}^{0}(\mathcal{P} \mathbb{N}) \backslash \Pi_{2}^{0}(\mathcal{P N})$.

3. $\mathcal{P}_{<\omega} \mathbb{N}$ is Wadge hard for $\boldsymbol{\Sigma}_{2}^{0}\left(\mathbf{2}^{\omega}\right)$ and for $\boldsymbol{\Pi}_{1}^{0}(\mathcal{P} \mathbb{N})$.

4. $\mathcal{P}_{<\omega} \mathbb{N}$ Wadge reduces no nonempty $\Sigma_{1}^{0}(\mathcal{P} \mathbb{N})$ family.

5. No subfamily of $\mathcal{P}_{<\omega} \mathbb{N}$ is Wadge hard for $\Sigma_{2}^{0}(\mathcal{P} \mathbb{N})$.

Items 3, 4 and 5 hold with effective versions. 
Proof.

1. Classical result (Kechris 1995), $\theta(\mathcal{P N})$ is just the set of all sequences eventually equal to 0 .

2. We use an argument from Selivanov (2005b). If $\mathcal{P}_{<\omega} \mathbb{N}$ were $\Pi_{2}^{0}(\mathcal{P} \mathbb{N})$ then the family of infinite sets would be $\Sigma_{2}^{0}(\mathcal{P} \mathbb{N})$, hence a countable union of differences of open sets $\cup_{n \in \mathbb{N}} \mathcal{U}_{n} \backslash \mathcal{V}_{n}$. If $X \in \mathcal{U}_{n} \backslash \mathcal{V}_{n}$ then there exists $A$ finite such that $A \subset X$ and $A \in \mathcal{U}_{n}$. Since $X \notin \mathcal{V}_{n}$ which is an upset, also $A \notin \mathcal{V}_{n}$. Thus, $\mathcal{U}_{n} \backslash \mathcal{V}_{n}$ contains a finite set, contradiction.

3. That $\mathcal{P}_{<\omega} \mathbb{N}$ is Wadge hard for $\boldsymbol{\Sigma}_{2}^{0}\left(\mathbf{2}^{\omega}\right)$ follows easily from the fact that the set of sequences eventually equal to 0 is $\boldsymbol{\Sigma}_{2}^{0}\left(\mathbf{2}^{\omega}\right)$-complete, and the continuity of the map $\theta^{-1}: \mathbf{2}^{\omega} \rightarrow \mathcal{P} \mathbb{N}$ (indeed it is an effective map). For the second assertion, any family $\mathcal{Y}$ in $\Pi_{1}^{0}(\mathcal{P} \mathbb{N})$ can be written $\mathcal{Y}=\{Z \mid \forall A \in \mathcal{A} A \nsubseteq Z\}$ where $\mathcal{A}$ is a subfamily of $\mathcal{P}_{<\omega} \mathbb{N}$. Define $f: \mathcal{P} \rightarrow \mathcal{P}$ such that $f(Z)=\mathbb{N}$ if $Z$ contains some $A \in \mathcal{A}$ and $f(Z)=\varnothing$, otherwise. Then, $f$ is a reduction of $\mathcal{X}$ to $\mathcal{P}_{<\omega} \mathbb{N}$.

4. Since $\mathcal{P}_{<\omega} \mathbb{N}$ is closed under subsets, and continuous reductions $\mathcal{P} \rightarrow \mathcal{N}$ are increasing, every family $\mathcal{X} \subset \mathcal{P} \mathbb{N}$ which is Wadge reducible to $\mathcal{P}_{<\omega} \mathbb{N}$ is closed under subsets. In particular, no nonempty $\Sigma_{1}^{0}(\mathcal{P} \mathbb{N})$ family admits a continuous reduction to $\mathcal{P}_{<\omega} \mathbb{N}$.

5. Observe that $\mathcal{P}_{<\omega} \mathbb{N}$ contains only chains of length $\omega$ and apply Proposition 5.8.

\section{Acknowledgements}

The authors are indebted to an anonymous referee for a huge number of improvements in our original manuscript.

\section{References}

Abramsky, S. and Jung, A. (1994) Domain theory. In: Gabbay, A and Maibaum (eds.) Handbook of Logic in Computer Science, volume 3,

Oxford University Press.

Becher, V. and Grigorieff, S. (2004) Recursion and topology on $\mathbf{2}^{\leq \omega}$ for possibly infinite computations. Theoretical Computer Science 322 (1) 85-136.

Becher, V. and Grigorieff, S. (2005) Random reals and possibly infinite computations. part I: Randomness in $\varnothing^{\prime}$. Journal of Symbolic Logic 70 (3) 891-913.

Becher, V. and Grigorieff, S. (2009) From index sets to randomness in $\varnothing^{n}$. Random reals and possibly infinite computations, part II. Journal of Symbolic Logic 74 (1) 124-156.

Becher, V. and Grigorieff, S. Borel and Hausdorff hierarchies in topological spaces of Choquet games and their effectivization. This volume.

Brattka, V. and Hertling, P. (1994) Continuity and computability of relations. Informatif Berichte 164, Fern Universität Gesamthochschule in Hagen, Fachbereich Informatik.

de Brecht, M. (2011) Quasi-Polish spaces. ArXiv:1108.1445v1, 40 pages.

Edalat, A. (1997) Domains for computation in mathematics, physics and exact real aritmetic. Bulletin of Symbolic Logic 3 (4) 401-452.

Ershov, Y. (1968) On a hierarchy of sets II. Algebra and Logic 7 (4) 15-47. 
Fokina, E. B., Friedman, S.-D. and Tornquist, A. (2010) The effective theory of Borel equivalence relations. Annals of Pure and Applied Logic 161 (7) 837-850.

Gierz, G., Hofmann, K. H., Keimel, K., Lawson, J. D., Mislove, M. and Scott, D. S. (2003) Continuous Lattices and Domains, Cambridge University Press.

Hertling, P. (1996a) Unstetigkeitgrade von Funktionen in der effektiven Analysis, Ph.D. thesis, Fern Universität Gesamthochschule in Hagen.

Hertling, P. (1996b) Topological complexity with continuous operations. Journal of Complexity 12 (4) 315-338.

Ikegami, D. (2010) Games in Set Theory and Logic, Ph.D. thesis, University of Amsterdam.

Ikegami, D., Schlicht, P. and Tanaka, H. (2012) Continuous reducibility for the real line, preprint, available at http://www.math.uni-bonn.de/people/schlicht/Ikegami\%20Schlicht $\% 20$ Tanaka\% 202012\%20linespread.pdf

Kechris, A. S. (1995) Classical Descriptive Set Theory, Graduate Texts in Mathematics, Springer.

Kuratowski, K. (1966) Topology, volume 1, Academic Press.

Marker, D. (2002) Lecture notes on Descriptive Set Theory. On Marker's home page, http://homepages.math.uic.edu/ marker/math512/dst.ps.

Moschovakis, Y. (1979/2009) Descriptive Set Theory, volume 155, American Mathematical Society.

Rogers, H. (1967) Theory of recursive functions and effective computability, McGraw-Hill.

Schlicht, P. (2012) Continuous reducibility and dimension, available at http://www.math.uni-bonn.de/ people/schlicht/Continuous\%20reducibility\%20and\%20dimension/crd.pdf

Selivanov, V. L. (2003) Wadge degrees of $\omega$-languages of deterministic Turing machines. Theoretical Informatics and Applications 37 (1) 67-83.

Selivanov, V. L. (2003) Extended abstract in STACS 2003 Proceedings. Lecture Notes in Computer Science 2607 97-108.

Selivanov, V. L. (2005) Variations on Wadge reducibility. Siberian Advances in Mathematics 15 (3) 44-80. (Also in Sixth Int. Workshop on Computability and Complexity in Analysis, Informatik Berichte, Uni-Hagen, 320-8/2004, 145-156.)

Selivanov, V. L. (2005a). Variations on Wadge reducibility. In: Proceedings of the 6th Workshop on Computability and Complexity in Analysis (CCA 2004). Electronic Notes in Theoretical Computer Science 120 159-171.

Selivanov, V. L. (2005b). Hierarchies in $\varphi$-spaces and applications. Mathematical Logic Quarterly 51 (1) 45-61.

Selivanov, V. L. (2006) Towards a descriptive set theory for domain-like structures. Theoretical Computer Science 365 (3) 258-282.

Selivanov, V. L. (2007) Hierarchies of $\Delta_{2}^{0}$-measurable k-partitions. Mathematical Logic Quarterly 53 (4-5) 446-461.

Selivanov, V. L. (2008) On the difference hierarchy in countably based $\mathrm{T}_{0}$-spaces. Electronic Notes in Theoretical Computer Science 221 257-269.

Tang, A. (1979) Chain properties in P $\omega$. Theoretical Computer Science 9 (2) 153-172.

Tang, A. (1981) Wadge reducibility and hausdorff difference hierarchy in $P \omega$. Lectures Notes in Mathematics 871 360-371.

Wadge, W. W. (1972) Degrees of complexity of subsets of the Baire space. Notices of the American Mathematical Society A-714.

Weihrauch, K. (2000) Computable Analysis. An Introduction, Springer. 